CRYSTALLOGRAPHIC COMMUNICATIONS

ISSN 2056-9890

Received 6 May 2015

Accepted 11 May 2015

Edited by J. Simpson, University of Otago, New Zealand

Keywords: crystal structure; tryptamine salts; phenoxyacetic acids; herbicides; 2,4-D; 3,5-D; hydrogen bonding

CCDC references: $1400285 ; 1400284$ Supporting information: this article has supporting information at journals.iucr.org/e

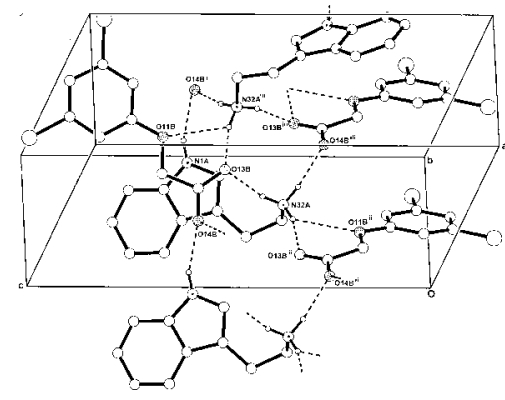

OPEN $\odot$ ACCESS

\section{Crystal structures and hydrogen bonding in the anhydrous tryptaminium salts of the isomeric (2,4-dichlorophenoxy)acetic and (3,5-dichloro- phenoxy)acetic acids}

\author{
Graham Smith $^{\mathrm{a} *}$ and Daniel E. Lynch ${ }^{\mathrm{b}}$ \\ ascience and Engineering Faculty, Queensland University of Technology, GPO Box 2434, Brisbane, Queensland 4001, \\ Australia, and ${ }^{\mathbf{b}}$ Exilica Ltd., The Technocentre, Puma Way, Coventry CV1 2TT, England. *Correspondence e-mail: \\ g.smith@qut.edu.au
}

The anhydrous salts of 2-( $1 H$-indol-3-yl)ethanamine (tryptamine) with isomeric (2,4-dichlorophenoxy)acetic acid (2,4-D) and (3,5-dichlorophenoxy)acetic (3,5-D), both $\mathrm{C}_{10} \mathrm{H}_{13} \mathrm{~N}_{2}{ }^{+} \cdot \mathrm{C}_{8} \mathrm{H}_{5} \mathrm{Cl}_{2} \mathrm{O}_{3}{ }^{-}$[(I) and (II), respectively], have been determined and their one-dimensional hydrogen-bonded polymeric structures are described. In the crystal of (I), the aminium $\mathrm{H}$ atoms are involved in three separate inter-species $\mathrm{N}-\mathrm{H} \cdots \mathrm{O}$ hydrogen-bonding interactions, two with carboxylate $\mathrm{O}$-atom acceptors and the third in an asymmetric three-centre bidentate carboxylate $O, O^{\prime}$ chelate [graph set $R_{1}^{2}(4)$ ]. The indole $\mathrm{H}$ atom forms an $\mathrm{N}-\mathrm{H} \cdots \mathrm{O}_{\text {carboxylate }}$ hydrogen bond, extending the chain structure along the $b$-axis direction. In (II), two of the three aminium $\mathrm{H}$ atoms are also involved in $\mathrm{N}-\mathrm{H} \cdots \mathrm{O}_{\text {carboxylate }}$ hydrogen bonds similar to (I) but with the third, a threecentre asymmetric interaction with carboxylate and phenoxy $\mathrm{O}$ atoms is found [graph set $R_{1}^{2}(5)$ ]. The chain polymeric extension is also along $b$. There are no $\pi-$ $\pi$ ring interactions in either of the structures. The aminium side-chain conformations differ significantly between the two structures, reflecting the conformational ambivalence of the tryptaminium cation, as found also in the benzoate salts.

\section{Chemical context}

2-(1H-Indol-3-yl)ethanamine (tryptamine) is an alkaloid found in plants and fungi and is a possible intermediate in the biosynthetic pathway to the plant hormone indole-3-acetic acid (Takahashi, 1986). It is also found in trace amounts in the mammalian brain, possibly acting as a neuromodulator or neurotransmitter (Jones, 1982). As a relatively strong base $\left(\mathrm{p} K_{\mathrm{a}}=10.2\right)$, it readily forms salts with a number of organic acids. To investigate the modes of hydrogen-bonding interaction in crystals of the tryptaminium salts of ring-substituted phenoxyacetic acid analogues, the reaction of tryptamine with two isomeric homologues, the herbicidally active (2,4-dichlorophenoxy)acetic acid (2,4-D) (Zumdahl, 2010) and (3,5dichlorophenoxy)acetic acid (3,5-D), gave the anhydrous salts, $\mathrm{C}_{10} \mathrm{H}_{13} \mathrm{~N}_{2}{ }^{+} \cdot \mathrm{C}_{8} \mathrm{H}_{5} \mathrm{Cl}_{2} \mathrm{O}_{3}{ }^{-}$, (I) and (II), respectively. Their structures and hydrogen-bonding modes are reported herein. The structure of the anhydrous salt with phenoxyacetic acid (Koshima et al., 1999) represents the only reported example of a salt from this acid series. In that crystal, chirality was generated through hydrogen bonding, giving cation-anion units related along a $2_{1}$ screw axes. A similar phenomenon was also observed in the tryptaminium 4-chlorobenzoate crystal (Koshima et al., 2005). 


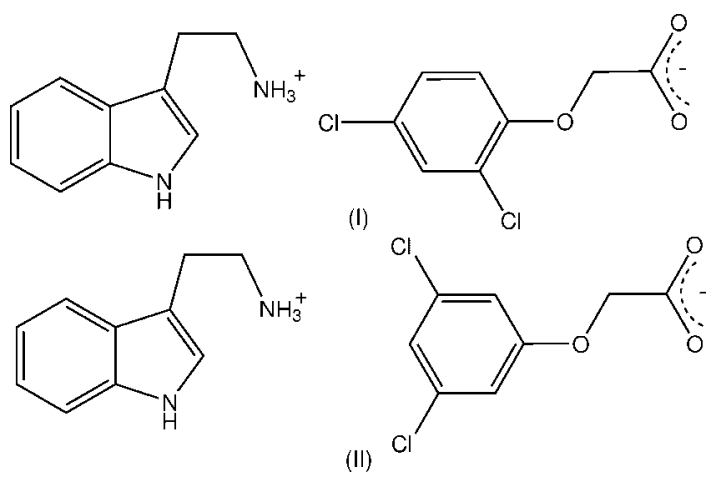

\section{Structural commentary}

The asymmetric units of (I) and (II) comprise a tryptaminium cation $(A)$ and either a 2,4-dichlorophenoxyacetate anion $(B)$ (I) (Fig. 1) or a (3,5-dichlorophenoxy)acetate anion (II) (Fig. 2). Unlike a number of tryptaminium salts of benzoic acids in which the benzene rings in the cation and anion species are essentially parallel, giving $\pi-\pi$ interactions, these planes in (I) and (II) are not so [dihedral angles $=74.1$ (3) and $24.68(17)^{\circ}$, respectively], giving no $\pi-\pi$ interactive effects.

The alkylaminium side chains in the cations of (I) and (II) differ significantly, with the torsion angles $\mathrm{C} 2 A-\mathrm{C} 3 A-$ $\mathrm{C} 31 A-\mathrm{C} 32 A$ and $\mathrm{C} 3 A-\mathrm{C} 31 A-\mathrm{C} 32 A-\mathrm{C} 32 A-\mathrm{N} 32 A$ being $-113.1(5), 58.6(5)^{\circ}$ in (I), $7.3(5)$ and in $75.7(4)^{\circ}$ (II), respectively. This variability is a standard feature in the structures of the known tryptaminium benzoate salts, which include the parent benzoate (Terakita et al., 2004), 4-chlorobenzoate (Koshima et al., 2005), 3,4-dimethoxybenzoate (Siripaisarnpipat \& Larsen, 1987), 3,5-dinitro-2-hydroxybenzoate (Lynch et al., 2015) and the pseudopolymorphic anhydrous, mono- and dihydrate 3,5-dinitrobenzoates salts (Lynch et al., 2015). In the structure of tryptamine, determined from powder diffraction data (Nowell et al., 2002), the corresponding angles are $-89.4(6)$ and $60.7(6)^{\circ}$.

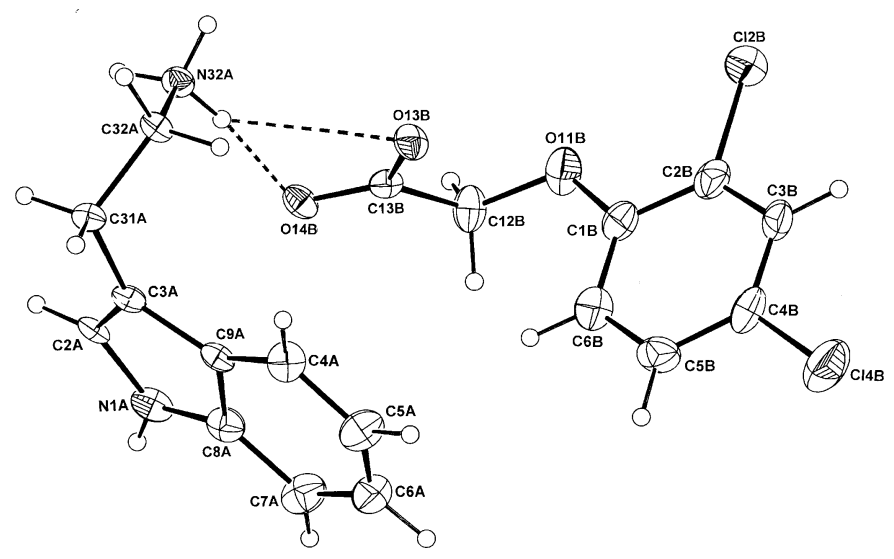

Figure 1

The atom-numbering scheme and the molecular conformation of the TRYP $^{+}$cation $(A)$ and the $2,4-\mathrm{D}^{-}$anion $(B)$ in (I) with displacement ellipsoids drawn at the $40 \%$ probability level. The cation-anion hydrogen bonds are shown as dashed lines.
Table 1

Hydrogen-bond geometry $\left(\AA,^{\circ}\right)$ for (I).

\begin{tabular}{lllll}
\hline$D-\mathrm{H} \cdots A$ & $D-\mathrm{H}$ & $\mathrm{H} \cdots A$ & $D \cdots A$ & $D-\mathrm{H} \cdots A$ \\
\hline $\mathrm{N} 1 A-\mathrm{H} 1 A \cdots \mathrm{O} 13 B^{\mathrm{i}}$ & $0.87(4)$ & $2.13(5)$ & $2.879(6)$ & $144(6)$ \\
$\mathrm{N} 32 A-\mathrm{H} 34 A \cdots \mathrm{O} 14 B^{\text {ii }}$ & $0.89(4)$ & $1.89(4)$ & $2.782(6)$ & $175(2)$ \\
$\mathrm{N} 32 A-\mathrm{H} 35 A \cdots \mathrm{O} 13 B^{\text {iii }}$ & $0.90(4)$ & $2.10(5)$ & $2.817(6)$ & $137(4)$ \\
$\mathrm{N} 32 A-\mathrm{H} 36 A \cdots \mathrm{O} 13 B$ & $0.89(3)$ & $2.57(4)$ & $3.231(6)$ & $132(4)$ \\
$\mathrm{N} 32 A-\mathrm{H} 36 A \cdots \mathrm{O} 14 B$ & $0.89(3)$ & $1.94(4)$ & $2.816(6)$ & $171(5)$ \\
\hline
\end{tabular}

Symmetry codes: (i) $x, y+1, z$; (ii) $-x+1, y-\frac{1}{2},-z+1$; (iii) $-x+1, y+\frac{1}{2},-z+1$.

In (I) the phenoxyacetate side chain of the 2,4-D anion is significantly rotated out of the benzene plane [defining torsion angle $\mathrm{C} 1 B-\mathrm{O} 11 B-\mathrm{C} 12 B-\mathrm{C} 13 B=81.2(6)^{\circ}$, similar to that of the parent acid which also has the synclinal side chain conformation (torsion angle $90 \pm 30^{\circ}$ ) (comparative torsion angle $=75.2^{\circ}$; Smith et al., 1976). However, in the potassium salt (Kennard et al., 1983) and the ammonium salt (Liu et al., 2009) (both hemihydrates), the antiperiplanar $\left(180 \pm 30^{\circ}\right)$ conformation is found. The 3,5-D anion in (II) adopts the antiperiplanar conformation with the defining $\mathrm{C} 1 B-\mathrm{O} 1 B-$ $\mathrm{C} 12 B-\mathrm{C} 13 B$ torsion angle $=-166.5$ (3). The structure of the parent acid is not known but the equivalent angle in the ammonium salt is $-171.35(15)^{\circ}$ (Smith, 2015) but in the 2:1 adduct of 3,5-D with 4,4'-bipyridine (Lynch et al., 2003), the angle is $-71.6(3)^{\circ}$ (synclinal).

\section{Supramolecular features}

In the crystal structures of (I) and (II), one-dimensional hydrogen-bonded structures involving $\mathrm{N}-\mathrm{H} \cdots \mathrm{O}_{\text {carboxylate }}$ interactions are found. However, the hydrogen-bonding patterns differ significantly. In the crystal of (I), the three aminium $\mathrm{H}$ atoms give different inter-species interactions, two with single carboxylate O-atom acceptors $\left(\mathrm{O} 13 B^{\mathrm{iii}}, \mathrm{O} 14 B^{\mathrm{ii}}\right)$ and third giving a three-centre $O, O^{\prime}$ chelate with carboxylate $\mathrm{O}$ atoms $(\mathrm{O} 13, \mathrm{O} 14)$ [graph set $R_{1}^{2}(4)$ ] (Table 1). The indole $\mathrm{H}$

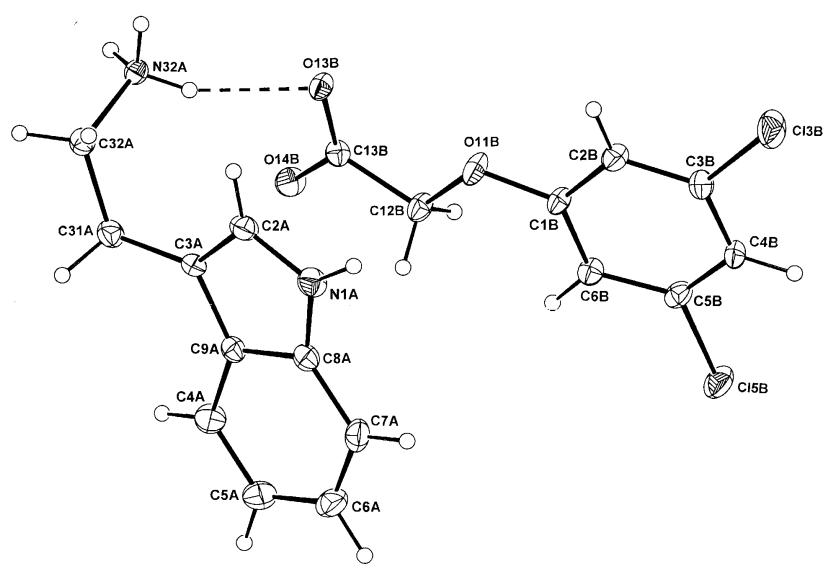

Figure 2

The atom-numbering scheme and the molecular conformation of the $\mathrm{TRYP}^{+}$cation $(A)$ and the $3,5-\mathrm{D}^{-}$anion $(B)$ in (II) with displacement ellipsoids drawn at the $40 \%$ probability level. The cation-anion hydrogen bond is shown as a dashed line. 


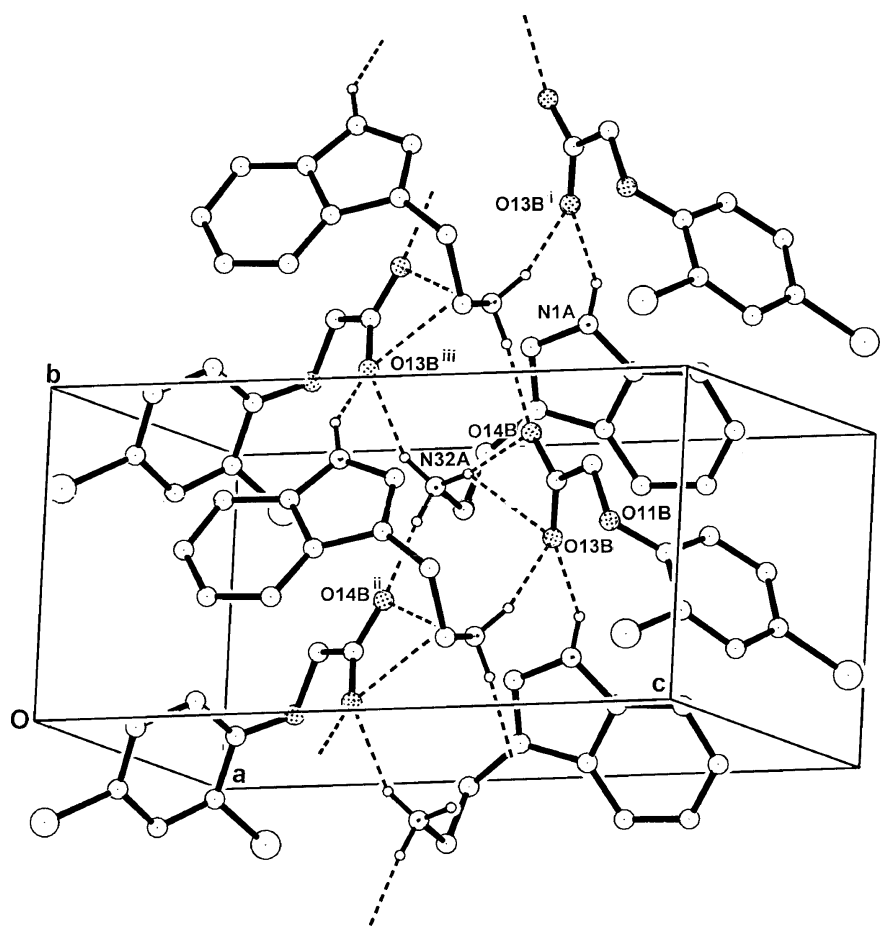

Figure 3

The one-dimensional hydrogen-bonded polymeric structure of (I) extending along [010], with non-associative $\mathrm{H}$ atoms omitted. For symmetry codes, see Table 1.

atom gives an $\mathrm{N}-\mathrm{H} \cdots \mathrm{O}_{\text {carboxylate }}$ hydrogen bond, extending the chain structure down the [010] axis (Fig. 3). In the crystal of (II), as with (I), two of the three aminium $\mathrm{N}-\mathrm{H} \cdots \mathrm{O}$ interactions are with single carboxylate $\mathrm{O}$ atoms [(O13B, $\mathrm{O} 14 B^{\mathrm{iii}}$ ) but the third differs in that it forms a three-centre asymmetric interaction with carboxylate and phenoxy $\mathrm{O}$ atoms of the anion $\left(\mathrm{O} 13 B^{\mathrm{ii}}, \mathrm{O} 11 B^{\mathrm{ii}}\right)$ [graph set $\left.R_{1}^{2}(4)\right]$ (Table 2$)$.

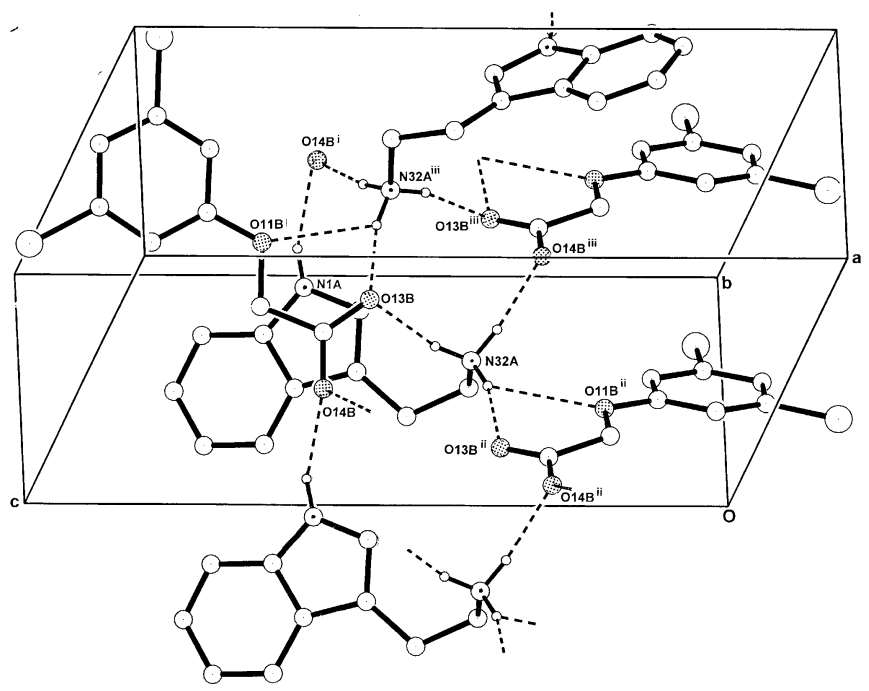

Figure 4

The one-dimensional hydrogen-bonded polymeric structure of (II) extending along [010], with non-associative H-atoms omitted. For symmetry codes, see Table 2.
Table 2

Hydrogen-bond geometry ( $\AA{ }^{\circ}$ ) for (II).

\begin{tabular}{lllll}
\hline$D-\mathrm{H} \cdots A$ & $D-\mathrm{H}$ & $\mathrm{H} \cdots A$ & $D \cdots A$ & $D-\mathrm{H} \cdots A$ \\
\hline $\mathrm{N} 1 A-\mathrm{H} 1 A \cdots \mathrm{O} 14 B^{\mathrm{i}}$ & $0.87(4)$ & $2.04(4)$ & $2.838(4)$ & $152(4)$ \\
$\mathrm{N} 32 A-\mathrm{H} 34 A \cdots \mathrm{O} 13 B$ & $0.87(2)$ & $2.05(3)$ & $2.875(4)$ & $160(4)$ \\
$\mathrm{N} 32 A-\mathrm{H} 35 A \cdots \mathrm{O} 11 B^{\text {ii }}$ & $0.89(3)$ & $2.60(4)$ & $3.160(4)$ & $122(3)$ \\
$\mathrm{N} 32 A-\mathrm{H} 35 A \cdots \mathrm{O} 13 B^{\text {ii }}$ & $0.89(3)$ & $1.87(3)$ & $2.739(4)$ & $164(4)$ \\
$\mathrm{N} 32 A-\mathrm{H} 36 A \cdots \mathrm{O} 14 B^{\text {iii }}$ & $0.89(4)$ & $1.90(4)$ & $2.775(4)$ & $170(4)$ \\
$\mathrm{C} 2 A-\mathrm{H} 2 A \cdots \mathrm{O} 13 B^{\text {iii }}$ & 0.95 & 2.55 & $3.495(4)$ & 177 \\
\hline
\end{tabular}

Symmetry codes: (i) $x, y+1, z$; (ii) $-x+1, y-\frac{1}{2},-z+1$; (iii) $-x+1, y+\frac{1}{2},-z+1$.

The chain polymeric $\mathrm{N} 1-\mathrm{H} \cdots \mathrm{O} 14 B$ extension is also along [010] (Fig. 4).

The present pair of structures of salts of tryptamine with isomeric (2,4-dichlorophenoxy)acetic acid and (3,5-dichlorophenoxy)acetic acid provide examples which further reflect the conformational ambivalence of the cationic alkylaminium side chain of the tryptamine cation, shown also in the benzoate salts.

\section{Synthesis and crystallization}

The title compounds (I) and (II) were prepared by warming together for $2 \mathrm{~min}$, solutions containing equimolar quantities of (2,4-dichlorophenoxy)acetic acid (2,4-D) or (3,5-dichlorophenoxy)acetic acid (3,5-D) (138 mg) with $100 \mathrm{mg}$ of tryptamine in ethanol. Room temperature evaporation of the solutions gave in both cases, colourless needles of (I) and (II) from which specimens were cleaved for the X-ray analyses.

\section{Refinement details}

Crystal data, data collection and structure refinement details are given in Table 3. Hydrogen atoms were placed in calculated positions $\left[\mathrm{C}-\mathrm{H}_{\text {aromatic }}=0.95 \AA\right.$ or $\mathrm{C}-\mathrm{H}_{\text {methylene }}=$ $0.99 \AA]$ and were allowed to ride in the refinements, with $U_{\text {iso }}(\mathrm{H})=1.2 U_{\text {eq }}(\mathrm{C})$. The aminium $\mathrm{H}$ atoms were located in difference-Fourier analyses and were allowed to refine with bond length restraints $[d(\mathrm{~N}-\mathrm{H}=0.88(2) \AA]$, and with $U_{\text {iso }}(\mathrm{H})=1.2 U_{\text {eq }}(\mathrm{N})$. Although possibly not of relevance in these crystals involving achiral molecules, the Flack absolute structure factors (Flack, 1983) were determined as 0.01 (7) for (II) (2232 Friedel pairs) and 0.45 (15) for (I) (1619 Friedel pairs), in the case of (I) suggesting possible racemic twinning. No indication of conventional twinning was found with the crystals of either isomer.

\section{Acknowledgements}

GS acknowledges financial support from the Science and Engineering Faculty, Queensland University of Technology.

\section{References}

Agilent (2013). CrysAlis PRO. Agilent Technologies Ltd., Yarnton, England.

Altomare, A., Cascarano, G., Giacovazzo, C. \& Guagliardi, A. (1993). J. Appl. Cryst. 26, 343-350. 
Table 3

Experimental details.

(I)

Crystal data

Chemical formula

$M_{\mathrm{r}}$

Crystal system, space group

Temperature (K)

$a, b, c(\AA)$

$\beta\left(^{\circ}\right)$

$V\left(\AA^{3}\right)$

$Z$

Radiation type

$\mu\left(\mathrm{mm}^{-1}\right)$

Crystal size (mm)

Data collection

Diffractometer

Absorption correction

$T_{\min }, T_{\max }$

No. of measured, independent and observed

$[I>2 \sigma(I)]$ reflections

$R_{\text {int }}$

$(\sin \theta / \lambda)_{\max }\left(\AA^{-1}\right)$

Refinement

$R\left[F^{2}>2 \sigma\left(F^{2}\right)\right], w R\left(F^{2}\right), S$

No. of reflections

No. of parameters

No. of restraints

$\mathrm{H}$-atom treatment

$\Delta \rho_{\max }, \Delta \rho_{\min }\left(\mathrm{e} \AA^{-3}\right)$

Absolute structure

Absolute structure parameter

\author{
$\mathrm{C}_{10} \mathrm{H}_{13} \mathrm{~N}_{2}{ }^{+} \cdot \mathrm{C}_{8} \mathrm{H}_{5} \mathrm{Cl}_{2} \mathrm{O}_{3}{ }^{-}$ \\ 381.25 \\ Monoclinic, $P 2_{1}$ \\ 200 \\ 8.9818 (11), 6.8899 (7), 14.6850 (15) \\ $93.565(9)$ \\ $907.00(17)$ \\ 2 \\ Mo $K \alpha$ \\ 0.38 \\ $0.50 \times 0.15 \times 0.05$
}

Oxford Diffraction Gemini-S CCD-detector

Multi-scan (CrysAlis PRO; Agilent, 2013)

$0.940,0.990$

3991, 2896, 2299

0.035

0.617

$0.068,0.187,1.06$

2896

226

1

$\mathrm{H}$ atoms treated by a mixture of indepen-

dent and constrained refinement

$0.37,-0.26$

Flack (1983)

$0.45(15)$
(II)

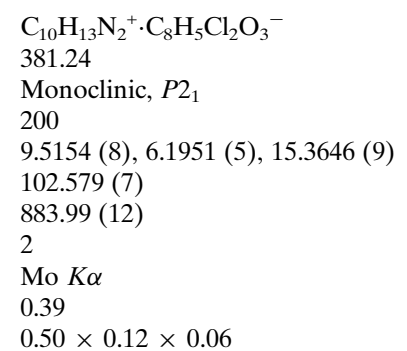

Oxford Diffraction Gemini-S CCD-detector Multi-scan (CrysAlis PRO; Agilent, 2013) $0.872,0.980$

$3845,2800,2451$

0.027

0.617

$0.042,0.105,1.08$

2800

238

5

$\mathrm{H}$ atoms treated by a mixture of indepen-

dent and constrained refinement $0.22,-0.24$

Flack (1983)

$0.01(7)$

Computer programs: CrysAlis PRO (Agilent, 2013), SIR92 (Altomare et al., 1993), SHELX97 (Sheldrick, 2008) within WinGX (Farrugia, 2012) and PLATON (Spek, 2009).

Farrugia, L. J. (2012). J. Appl. Cryst. 45, 849-854.

Flack, H. D. (1983). Acta Cryst. A39, 876-881.

Jones, R. S. G. (1982). Prog. Neurobiol. 19, 117-139.

Kennard, C. H. L., Smith, G. \& O'Reilly, E. J. (1983). Inorg. Chim. Acta, 77, L181-L184.

Koshima, H., Honke, S. \& Fujita, J. (1999). J. Org. Chem. 64, 39163921.

Koshima, H., Nagano, M. \& Asahi, T. (2005). J. Am. Chem. Soc. 127, 2455-2463.

Liu, H.-L., Guo, S.-H., Li, Y.-Y. \& Jian, F.-F. (2009). Acta Cryst. E65, o1905.

Lynch, D. E., Barfield, J., Frost, J., Antrobus, R. \& Simmons, J. (2003). Cryst. Eng. 6, 109-122.

Lynch, D. E., Cox, M. \& Smith, G. (2015). Dyes Pigments, doi: 10.1016/j. dyepig. 2015.01.028.
Nowell, H., Attfield, J. P. \& Cole, J. C. (2002). Acta Cryst. B58, 835840.

Sheldrick, G. M. (2008). Acta Cryst. A64, 112-122.

Siripaisarnpipat, S. \& Larsen, S. (1987). Acta Chem. Scand. Ser. A, 41, 539-547.

Smith, G. (2015). Unpublished results.

Smith, G., Kennard, C. H. L. \& White, A. H. (1976). J. Chem. Soc. Perkin Trans. 2, pp. 791-792.

Spek, A. L. (2009). Acta Cryst. D65, 148-155.

Takahashi, N. (1986). In Chemistry of Plant Hormones. Florida: CRC Press.

Terakita, A., Matsunaga, H., Ueda, T., Eguchi, T., Echigoya, M., Umemoto, K. \& Godo, M. (2004). Chem. Pharm. Bull. 52, 546-551.

Zumdahl, R. L. (2010). In A History of Weed Science in the United States. New York: Elsevier. 


\section{supporting information}

Acta Cryst. (2015). E71, 671-674 [doi:10.1107/S205698901500907X]

\section{Crystal structures and hydrogen bonding in the anhydrous tryptaminium salts of the isomeric (2,4-dichlorophenoxy)acetic and (3,5-dichlorophenoxy)acetic acids}

\section{Graham Smith and Daniel E. Lynch}

\section{Computing details}

For both compounds, data collection: CrysAlis PRO (Agilent, 2013); cell refinement: CrysAlis PRO (Agilent, 2013); data reduction: CrysAlis PRO (Agilent, 2013); program(s) used to solve structure: SIR92 (Altomare et al., 1993). Program(s) used to refine structure: SHELX97 (Sheldrick, 2008) within WinGX (Farrugia, 2012) for (I); SHELXL97 (Sheldrick, 2008) within WinGX (Farrugia, 2012) for (II). For both compounds, molecular graphics: PLATON (Spek, 2009); software used to prepare material for publication: PLATON (Spek, 2009).

\section{(I) 2-(1H-Indol-3-yl)ethanaminium (2,4-dichlorophenoxy)acetate}

\section{Crystal data}

$\mathrm{C}_{10} \mathrm{H}_{13} \mathrm{~N}_{2}{ }^{+} \cdot \mathrm{C}_{8} \mathrm{H}_{5} \mathrm{Cl}_{2} \mathrm{O}_{3}^{-}$

$M_{r}=381.25$

Monoclinic, $P 2_{1}$

Hall symbol: $\mathrm{P} 2 \mathrm{yb}$

$a=8.9818(11) \AA$

$b=6.8899(7) \AA$

$c=14.6850(15) \AA$

$\beta=93.565(9)^{\circ}$

$V=907.00(17) \AA^{3}$

$Z=2$

\section{Data collection}

Oxford Diffraction Gemini-S CCD-detector diffractometer

Radiation source: Enhance (Mo) X-ray source

Graphite monochromator

Detector resolution: 16.077 pixels $\mathrm{mm}^{-1}$

$\omega$ scans

Absorption correction: multi-scan

(CrysAlis PRO; Agilent, 2013)

$T_{\min }=0.940, T_{\max }=0.990$

Refinement

Refinement on $F^{2}$

Least-squares matrix: full

$R\left[F^{2}>2 \sigma\left(F^{2}\right)\right]=0.068$

$w R\left(F^{2}\right)=0.187$

$S=1.06$
$F(000)=396$

$D_{\mathrm{x}}=1.396 \mathrm{Mg} \mathrm{m}^{-3}$

Mo $K \alpha$ radiation, $\lambda=0.71073 \AA$

Cell parameters from 940 reflections

$\theta=3.8-24.0^{\circ}$

$\mu=0.38 \mathrm{~mm}^{-1}$

$T=200 \mathrm{~K}$

Needle, colourless

$0.50 \times 0.15 \times 0.05 \mathrm{~mm}$

3991 measured reflections

2896 independent reflections

2299 reflections with $I>2 \sigma(I)$

$R_{\text {int }}=0.035$

$\theta_{\max }=26.0^{\circ}, \theta_{\min }=3.3^{\circ}$

$h=-10 \rightarrow 11$

$k=-7 \rightarrow 8$

$l=-17 \rightarrow 18$

2896 reflections

226 parameters

1 restraint

Primary atom site location: structure-invariant direct methods 
Secondary atom site location: difference Fourier map

Hydrogen site location: inferred from neighbouring sites

$\mathrm{H}$ atoms treated by a mixture of independent and constrained refinement

$$
\begin{aligned}
& w=1 /\left[\sigma^{2}\left(F_{\mathrm{o}}^{2}\right)+(0.0918 P)^{2}+0.3461 P\right] \\
& \text { where } P=\left(F_{\mathrm{o}}^{2}+2 F_{\mathrm{c}}^{2}\right) / 3 \\
& (\Delta / \sigma)_{\max }<0.001 \\
& \Delta \rho_{\max }=0.37 \text { e } \AA^{-3} \\
& \Delta \rho_{\min }=-0.26 \mathrm{e} \AA^{-3}
\end{aligned}
$$

Absolute structure: Flack (1983)

Absolute structure parameter: 0.45 (15)

Special details

Geometry. Bond distances, angles etc. have been calculated using the rounded fractional coordinates. All su's are estimated from the variances of the (full) variance-covariance matrix. The cell esds are taken into account in the estimation of distances, angles and torsion angles

Refinement. Refinement of $\mathrm{F}^{2}$ against ALL reflections. The weighted R-factor wR and goodness of fit $\mathrm{S}$ are based on $\mathrm{F}^{2}$,

\begin{tabular}{|c|c|c|c|c|}
\hline & $x$ & $y$ & $z$ & $U_{\text {iso }} * / U_{\text {eq }}$ \\
\hline $\mathrm{Cl} 2 \mathrm{~B}$ & 0.28249 (19) & 0.2639 & $0.84021(11)$ & $0.0625(6)$ \\
\hline Cl4B & $0.8117(2)$ & $0.2352(4)$ & $1.03185(12)$ & $0.0817(8)$ \\
\hline O11B & $0.3858(5)$ & $0.6285(7)$ & $0.7750(3)$ & $0.0565(16)$ \\
\hline O13B & $0.5619(4)$ & $0.6213(5)$ & 0.6335 & $0.0378(12)$ \\
\hline O14B & $0.5207(4)$ & $0.9327(5)$ & 0.6033 & $0.0346(11)$ \\
\hline $\mathrm{C} 1 \mathrm{~B}$ & $0.4889(7)$ & $0.5483(10)$ & $0.8340(4)$ & 0.0444 (19) \\
\hline $\mathrm{C} 2 \mathrm{~B}$ & $0.4569(7)$ & $0.3677(10)$ & $0.8717(4)$ & $0.047(2)$ \\
\hline C3B & $0.5495(7)$ & $0.2673(11)$ & $0.9322(3)$ & 0.049 (2) \\
\hline C4B & $0.6859(8)$ & $0.3564(12)$ & $0.9564(4)$ & $0.057(3)$ \\
\hline C5B & $0.7253(7)$ & $0.5320(11)$ & $0.9216(4)$ & $0.051(2)$ \\
\hline C6B & $0.6281(8)$ & $0.6256(11)$ & 0.8585 (4) & $0.055(2)$ \\
\hline C12B & $0.4160(7)$ & $0.8064(9)$ & $0.7333(4)$ & $0.045(2)$ \\
\hline C13B & $0.5129(5)$ & $0.7839(8)$ & 0.6498 & $0.0290(17)$ \\
\hline N1A & $0.7673(5)$ & $1.3071(7)$ & $0.6100(3)$ & $0.0397(16)$ \\
\hline N32A & $0.6027(5)$ & $0.7972(7)$ & $0.4326(3)$ & $0.0317(14)$ \\
\hline $\mathrm{C} 2 \mathrm{~A}$ & $0.7670(5)$ & $1.2445(7)$ & 0.5215 & $0.0262(14)$ \\
\hline $\mathrm{C} 3 \mathrm{~A}$ & $0.8329(5)$ & $1.0707(7)$ & $0.5166(3)$ & $0.0274(16)$ \\
\hline $\mathrm{C} 4 \mathrm{~A}$ & $0.9521(6)$ & $0.8575(9)$ & $0.6506(4)$ & $0.0410(17)$ \\
\hline $\mathrm{C} 5 \mathrm{~A}$ & $0.9790(7)$ & $0.8583(11)$ & $0.7434(4)$ & $0.052(2)$ \\
\hline C6A & $0.9345(8)$ & $1.0100(12)$ & $0.7973(5)$ & $0.060(3)$ \\
\hline C7A & $0.8638(7)$ & $1.1725(11)$ & $0.7595(4)$ & $0.055(2)$ \\
\hline $\mathrm{C} 8 \mathrm{~A}$ & $0.8359(6)$ & $1.1754(9)$ & $0.6647(4)$ & 0.0388 (19) \\
\hline C9A & $0.8793(5)$ & $1.0155(8)$ & $0.6100(4)$ & $0.0296(16)$ \\
\hline C31A & $0.8527(6)$ & $0.9501(7)$ & 0.4333 & $0.0304(17)$ \\
\hline C32A & $0.7656(5)$ & $0.7611(8)$ & 0.4290 & $0.0296(16)$ \\
\hline H3B & 0.52290 & 0.14500 & 0.95620 & $0.0580^{*}$ \\
\hline H5B & 0.81840 & 0.58950 & 0.94030 & $0.0620^{*}$ \\
\hline H6B & 0.65760 & 0.74420 & 0.83190 & $0.0660 *$ \\
\hline H12B & 0.46840 & 0.89250 & 0.77880 & $0.0550 *$ \\
\hline
\end{tabular}
conventional R-factors $R$ are based on $F$, with $F$ set to zero for negative $F^{2}$. The threshold expression of $F^{2}>2 \operatorname{sigma}\left(F^{2}\right)$ is used only for calculating R-factors (gt) etc. and is not relevant to the choice of reflections for refinement. R-factors based on $\mathrm{F}^{2}$ are statistically about twice as large as those based on F, and R-factors based on ALL data will be even larger.

Fractional atomic coordinates and isotropic or equivalent isotropic displacement parameters $\left(\AA^{2}\right)$ 


\begin{tabular}{lllll} 
H13B & 0.32040 & 0.86940 & 0.71320 & $0.0550^{*}$ \\
H1A & $0.743(7)$ & $1.423(5)$ & $0.627(4)$ & $0.0480^{*}$ \\
H2A & 0.72550 & 1.31490 & 0.47040 & $0.0310^{*}$ \\
H4A & 0.98260 & 0.75130 & 0.61500 & $0.0490^{*}$ \\
H5A & 1.02980 & 0.75130 & 0.77180 & $0.0620^{*}$ \\
H6A & 0.95280 & 1.00250 & 0.86160 & $0.0720^{*}$ \\
H7A & 0.83540 & 1.27770 & 0.79650 & $0.0660^{*}$ \\
H30B & 0.96010 & 0.91970 & 0.43050 & $0.0360^{*}$ \\
H31B & 0.82230 & 1.02830 & 0.37880 & $0.0360^{*}$ \\
H32B & 0.78460 & 0.69190 & 0.37180 & $0.0360^{*}$ \\
H33B & 0.80010 & 0.67720 & 0.48090 & $0.0360^{*}$ \\
H34A & $0.559(6)$ & $0.684(5)$ & $0.419(3)$ & $0.0380^{*}$ \\
H35A & $0.581(6)$ & $0.883(6)$ & $0.388(3)$ & $0.0380^{*}$ \\
H36A & $0.581(6)$ & $0.828(8)$ & $0.4889(19)$ & $0.0380^{*}$ \\
\hline
\end{tabular}

Atomic displacement parameters $\left(\AA^{2}\right)$

\begin{tabular}{lllllll}
\hline & $U^{11}$ & $U^{22}$ & $U^{33}$ & $U^{12}$ & $U^{13}$ & $U^{23}$ \\
\hline C12B & $0.0639(11)$ & $0.0664(11)$ & $0.0572(10)$ & $-0.0152(10)$ & $0.0030(7)$ & $-0.0014(9)$ \\
C14B & $0.0779(13)$ & $0.1142(19)$ & $0.0512(10)$ & $0.0072(13)$ & $-0.0110(8)$ & $0.0160(11)$ \\
O11B & $0.055(3)$ & $0.064(3)$ & $0.051(2)$ & $0.004(2)$ & $0.007(2)$ & $0.017(2)$ \\
O13B & $0.044(2)$ & $0.024(2)$ & $0.045(2)$ & $0.0037(17)$ & $-0.0007(17)$ & $0.0043(16)$ \\
O14B & $0.0250(19)$ & $0.029(2)$ & $0.050(2)$ & $-0.0007(16)$ & $0.0030(15)$ & $0.0017(17)$ \\
C1B & $0.047(3)$ & $0.055(4)$ & $0.032(3)$ & $0.005(3)$ & $0.009(2)$ & $-0.002(3)$ \\
C2B & $0.063(4)$ & $0.050(4)$ & $0.029(3)$ & $0.004(3)$ & $0.009(3)$ & $-0.005(3)$ \\
C3B & $0.069(4)$ & $0.049(4)$ & $0.029(3)$ & $-0.005(3)$ & $0.011(2)$ & $0.004(3)$ \\
C4B & $0.069(5)$ & $0.074(5)$ & $0.028(3)$ & $0.007(4)$ & $0.012(3)$ & $0.005(3)$ \\
C5B & $0.036(3)$ & $0.064(5)$ & $0.054(4)$ & $-0.010(3)$ & $0.004(3)$ & $-0.008(4)$ \\
C6B & $0.059(4)$ & $0.059(4)$ & $0.047(4)$ & $0.000(4)$ & $0.008(3)$ & $0.004(3)$ \\
C12B & $0.048(4)$ & $0.042(4)$ & $0.047(3)$ & $0.016(3)$ & $0.010(3)$ & $0.015(3)$ \\
C13B & $0.028(3)$ & $0.025(3)$ & $0.033(3)$ & $0.006(2)$ & $-0.0060(18)$ & $-0.002(2)$ \\
N1A & $0.031(2)$ & $0.028(3)$ & $0.060(3)$ & $0.004(2)$ & $0.003(2)$ & $-0.009(2)$ \\
N32A & $0.024(2)$ & $0.035(3)$ & $0.036(2)$ & $-0.003(2)$ & $0.0010(17)$ & $0.000(2)$ \\
C2A & $0.019(2)$ & $0.016(2)$ & $0.043(3)$ & $-0.004(2)$ & $-0.0016(18)$ & $0.001(2)$ \\
C3A & $0.018(2)$ & $0.023(3)$ & $0.041(3)$ & $0.000(2)$ & $0.0008(19)$ & $-0.004(2)$ \\
C4A & $0.030(3)$ & $0.043(3)$ & $0.050(3)$ & $0.013(3)$ & $0.003(2)$ & $0.003(3)$ \\
C5A & $0.042(3)$ & $0.062(4)$ & $0.050(4)$ & $0.013(3)$ & $-0.008(3)$ & $0.000(3)$ \\
C6A & $0.068(5)$ & $0.072(5)$ & $0.041(4)$ & $-0.016(4)$ & $0.003(3)$ & $-0.002(4)$ \\
C7A & $0.045(4)$ & $0.075(5)$ & $0.045(3)$ & $0.003(4)$ & $0.009(3)$ & $-0.012(3)$ \\
C8A & $0.027(3)$ & $0.044(4)$ & $0.046(3)$ & $-0.002(3)$ & $0.008(2)$ & $-0.005(3)$ \\
C9A & $0.020(2)$ & $0.027(3)$ & $0.042(3)$ & $-0.005(2)$ & $0.0047(19)$ & $-0.003(2)$ \\
C31A & $0.026(3)$ & $0.030(3)$ & $0.035(3)$ & $-0.002(2)$ & $0.001(2)$ & $-0.004(2)$ \\
C32A & $0.025(2)$ & $0.023(3)$ & $0.041(3)$ & $-0.001(2)$ & $0.0029(19)$ & $-0.001(2)$ \\
& & & & & & \\
\hline
\end{tabular}

Geometric parameters $\left(\AA,^{\circ}\right)$

\begin{tabular}{llll}
\hline $\mathrm{C} 2 \mathrm{~B}-\mathrm{C} 2 \mathrm{~B}$ & $1.758(7)$ & $\mathrm{C} 6 \mathrm{~B}-\mathrm{H} 6 \mathrm{~B}$ & 0.9500 \\
$\mathrm{C} 14 \mathrm{~B}-\mathrm{C} 4 \mathrm{~B}$ & $1.745(7)$ & $\mathrm{C} 12 \mathrm{~B}-\mathrm{H} 13 \mathrm{~B}$ & 0.9900
\end{tabular}




\begin{tabular}{|c|c|c|c|}
\hline $\mathrm{O} 11 \mathrm{~B}-\mathrm{C} 1 \mathrm{~B}$ & $1.347(8)$ & $\mathrm{C} 12 \mathrm{~B}-\mathrm{H} 12 \mathrm{~B}$ & 0.9900 \\
\hline $\mathrm{O} 11 \mathrm{~B}-\mathrm{C} 12 \mathrm{~B}$ & $1.404(8)$ & $\mathrm{C} 2 \mathrm{~A}-\mathrm{C} 3 \mathrm{~A}$ & $1.340(7)$ \\
\hline $\mathrm{O} 13 \mathrm{~B}-\mathrm{C} 13 \mathrm{~B}$ & $1.233(6)$ & $\mathrm{C} 3 \mathrm{~A}-\mathrm{C} 9 \mathrm{~A}$ & $1.459(7)$ \\
\hline $\mathrm{O} 14 \mathrm{~B}-\mathrm{C} 13 \mathrm{~B}$ & $1.236(6)$ & $\mathrm{C} 3 \mathrm{~A}-\mathrm{C} 31 \mathrm{~A}$ & $1.499(6)$ \\
\hline $\mathrm{N} 1 \mathrm{~A}-\mathrm{C} 8 \mathrm{~A}$ & $1.337(8)$ & $\mathrm{C} 4 \mathrm{~A}-\mathrm{C} 5 \mathrm{~A}$ & $1.369(8)$ \\
\hline $\mathrm{N} 1 \mathrm{~A}-\mathrm{C} 2 \mathrm{~A}$ & $1.369(6)$ & $\mathrm{C} 4 \mathrm{~A}-\mathrm{C} 9 \mathrm{~A}$ & $1.386(8)$ \\
\hline $\mathrm{N} 32 \mathrm{~A}-\mathrm{C} 32 \mathrm{~A}$ & $1.488(6)$ & $\mathrm{C} 5 \mathrm{~A}-\mathrm{C} 6 \mathrm{~A}$ & $1.385(10)$ \\
\hline N1A-H1A & $0.87(4)$ & $\mathrm{C} 6 \mathrm{~A}-\mathrm{C} 7 \mathrm{~A}$ & $1.386(11)$ \\
\hline $\mathrm{N} 32 \mathrm{~A}-\mathrm{H} 34 \mathrm{~A}$ & $0.89(4)$ & $\mathrm{C} 7 \mathrm{~A}-\mathrm{C} 8 \mathrm{~A}$ & $1.399(8)$ \\
\hline $\mathrm{N} 32 \mathrm{~A}-\mathrm{H} 36 \mathrm{~A}$ & $0.89(3)$ & $\mathrm{C} 8 \mathrm{~A}-\mathrm{C} 9 \mathrm{~A}$ & $1.432(8)$ \\
\hline $\mathrm{N} 32 \mathrm{~A}-\mathrm{H} 35 \mathrm{~A}$ & $0.90(4)$ & $\mathrm{C} 31 \mathrm{~A}-\mathrm{C} 32 \mathrm{~A}$ & $1.518(7)$ \\
\hline $\mathrm{C} 1 \mathrm{~B}-\mathrm{C} 2 \mathrm{~B}$ & $1.399(9)$ & $\mathrm{C} 2 \mathrm{~A}-\mathrm{H} 2 \mathrm{~A}$ & 0.9500 \\
\hline $\mathrm{C} 1 \mathrm{~B}-\mathrm{C} 6 \mathrm{~B}$ & $1.386(10)$ & $\mathrm{C} 4 \mathrm{~A}-\mathrm{H} 4 \mathrm{~A}$ & 0.9500 \\
\hline $\mathrm{C} 2 \mathrm{~B}-\mathrm{C} 3 \mathrm{~B}$ & $1.367(9)$ & $\mathrm{C} 5 \mathrm{~A}-\mathrm{H} 5 \mathrm{~A}$ & 0.9500 \\
\hline $\mathrm{C} 3 \mathrm{~B}-\mathrm{C} 4 \mathrm{~B}$ & $1.396(10)$ & C6A-H6A & 0.9500 \\
\hline $\mathrm{C} 4 \mathrm{~B}-\mathrm{C} 5 \mathrm{~B}$ & $1.369(11)$ & $\mathrm{C} 7 \mathrm{~A}-\mathrm{H} 7 \mathrm{~A}$ & 0.9500 \\
\hline $\mathrm{C} 5 \mathrm{~B}-\mathrm{C} 6 \mathrm{~B}$ & $1.391(9)$ & $\mathrm{C} 31 \mathrm{~A}-\mathrm{H} 30 \mathrm{~B}$ & 0.9900 \\
\hline $\mathrm{C} 12 \mathrm{~B}-\mathrm{C} 13 \mathrm{~B}$ & $1.555(7)$ & $\mathrm{C} 31 \mathrm{~A}-\mathrm{H} 31 \mathrm{~B}$ & 0.9900 \\
\hline $\mathrm{C} 3 \mathrm{~B}-\mathrm{H} 3 \mathrm{~B}$ & 0.9500 & $\mathrm{C} 32 \mathrm{~A}-\mathrm{H} 32 \mathrm{~B}$ & 0.9900 \\
\hline $\mathrm{C} 5 \mathrm{~B}-\mathrm{H} 5 \mathrm{~B}$ & 0.9500 & $\mathrm{C} 32 \mathrm{~A}-\mathrm{H} 33 \mathrm{~B}$ & 0.9900 \\
\hline $\mathrm{C} 1 \mathrm{~B}-\mathrm{O} 11 \mathrm{~B}-\mathrm{C} 12 \mathrm{~B}$ & $119.7(5)$ & $\mathrm{N} 1 \mathrm{~A}-\mathrm{C} 2 \mathrm{~A}-\mathrm{C} 3 \mathrm{~A}$ & $111.0(4)$ \\
\hline $\mathrm{C} 2 \mathrm{~A}-\mathrm{N} 1 \mathrm{~A}-\mathrm{C} 8 \mathrm{~A}$ & $109.3(5)$ & $\mathrm{C} 2 \mathrm{~A}-\mathrm{C} 3 \mathrm{~A}-\mathrm{C} 9 \mathrm{~A}$ & $106.5(4)$ \\
\hline $\mathrm{C} 8 \mathrm{~A}-\mathrm{N} 1 \mathrm{~A}-\mathrm{H} 1 \mathrm{~A}$ & $125(4)$ & $\mathrm{C} 2 \mathrm{~A}-\mathrm{C} 3 \mathrm{~A}-\mathrm{C} 31 \mathrm{~A}$ & $127.9(4)$ \\
\hline $\mathrm{C} 2 \mathrm{~A}-\mathrm{N} 1 \mathrm{~A}-\mathrm{H} 1 \mathrm{~A}$ & $125(4)$ & $\mathrm{C} 9 \mathrm{~A}-\mathrm{C} 3 \mathrm{~A}-\mathrm{C} 31 \mathrm{~A}$ & $125.6(4)$ \\
\hline $\mathrm{C} 32 \mathrm{~A}-\mathrm{N} 32 \mathrm{~A}-\mathrm{H} 35 \mathrm{~A}$ & $105(3)$ & $\mathrm{C} 5 \mathrm{~A}-\mathrm{C} 4 \mathrm{~A}-\mathrm{C} 9 \mathrm{~A}$ & $118.3(6)$ \\
\hline $\mathrm{H} 34 \mathrm{~A}-\mathrm{N} 32 \mathrm{~A}-\mathrm{H} 36 \mathrm{~A}$ & $107(5)$ & $\mathrm{C} 4 \mathrm{~A}-\mathrm{C} 5 \mathrm{~A}-\mathrm{C} 6 \mathrm{~A}$ & $122.2(7)$ \\
\hline $\mathrm{C} 32 \mathrm{~A}-\mathrm{N} 32 \mathrm{~A}-\mathrm{H} 36 \mathrm{~A}$ & $110(3)$ & $\mathrm{C} 5 \mathrm{~A}-\mathrm{C} 6 \mathrm{~A}-\mathrm{C} 7 \mathrm{~A}$ & $121.5(6)$ \\
\hline $\mathrm{H} 34 \mathrm{~A}-\mathrm{N} 32 \mathrm{~A}-\mathrm{H} 35 \mathrm{~A}$ & $110(4)$ & $\mathrm{C} 6 \mathrm{~A}-\mathrm{C} 7 \mathrm{~A}-\mathrm{C} 8 \mathrm{~A}$ & $117.3(6)$ \\
\hline $\mathrm{C} 32 \mathrm{~A}-\mathrm{N} 32 \mathrm{~A}-\mathrm{H} 34 \mathrm{~A}$ & $105(3)$ & $\mathrm{N} 1 \mathrm{~A}-\mathrm{C} 8 \mathrm{~A}-\mathrm{C} 9 \mathrm{~A}$ & $108.5(5)$ \\
\hline $\mathrm{H} 35 \mathrm{~A}-\mathrm{N} 32 \mathrm{~A}-\mathrm{H} 36 \mathrm{~A}$ & $118(4)$ & $\mathrm{C} 7 \mathrm{~A}-\mathrm{C} 8 \mathrm{~A}-\mathrm{C} 9 \mathrm{~A}$ & $120.6(6)$ \\
\hline $\mathrm{O} 11 \mathrm{~B}-\mathrm{C} 1 \mathrm{~B}-\mathrm{C} 2 \mathrm{~B}$ & $118.0(6)$ & $\mathrm{N} 1 \mathrm{~A}-\mathrm{C} 8 \mathrm{~A}-\mathrm{C} 7 \mathrm{~A}$ & $130.9(6)$ \\
\hline $\mathrm{C} 2 \mathrm{~B}-\mathrm{C} 1 \mathrm{~B}-\mathrm{C} 6 \mathrm{~B}$ & $116.3(6)$ & $\mathrm{C} 3 \mathrm{~A}-\mathrm{C} 9 \mathrm{~A}-\mathrm{C} 4 \mathrm{~A}$ & $135.2(5)$ \\
\hline $\mathrm{O} 11 \mathrm{~B}-\mathrm{C} 1 \mathrm{~B}-\mathrm{C} 6 \mathrm{~B}$ & $125.6(6)$ & $\mathrm{C} 3 \mathrm{~A}-\mathrm{C} 9 \mathrm{~A}-\mathrm{C} 8 \mathrm{~A}$ & $104.8(5)$ \\
\hline $\mathrm{Cl} 2 \mathrm{~B}-\mathrm{C} 2 \mathrm{~B}-\mathrm{C} 1 \mathrm{~B}$ & $117.4(5)$ & $\mathrm{C} 4 \mathrm{~A}-\mathrm{C} 9 \mathrm{~A}-\mathrm{C} 8 \mathrm{~A}$ & $120.1(5)$ \\
\hline $\mathrm{C} 1 \mathrm{~B}-\mathrm{C} 2 \mathrm{~B}-\mathrm{C} 3 \mathrm{~B}$ & $125.2(6)$ & $\mathrm{C} 3 \mathrm{~A}-\mathrm{C} 31 \mathrm{~A}-\mathrm{C} 32 \mathrm{~A}$ & $115.0(4)$ \\
\hline $\mathrm{Cl} 2 \mathrm{~B}-\mathrm{C} 2 \mathrm{~B}-\mathrm{C} 3 \mathrm{~B}$ & $117.5(5)$ & $\mathrm{N} 32 \mathrm{~A}-\mathrm{C} 32 \mathrm{~A}-\mathrm{C} 31 \mathrm{~A}$ & $111.1(4)$ \\
\hline $\mathrm{C} 2 \mathrm{~B}-\mathrm{C} 3 \mathrm{~B}-\mathrm{C} 4 \mathrm{~B}$ & $115.6(6)$ & $\mathrm{N} 1 \mathrm{~A}-\mathrm{C} 2 \mathrm{~A}-\mathrm{H} 2 \mathrm{~A}$ & 125.00 \\
\hline $\mathrm{Cl} 4 \mathrm{~B}-\mathrm{C} 4 \mathrm{~B}-\mathrm{C} 5 \mathrm{~B}$ & $119.2(5)$ & $\mathrm{C} 3 \mathrm{~A}-\mathrm{C} 2 \mathrm{~A}-\mathrm{H} 2 \mathrm{~A}$ & 125.00 \\
\hline $\mathrm{Cl} 4 \mathrm{~B}-\mathrm{C} 4 \mathrm{~B}-\mathrm{C} 3 \mathrm{~B}$ & $118.3(6)$ & $\mathrm{C} 5 \mathrm{~A}-\mathrm{C} 4 \mathrm{~A}-\mathrm{H} 4 \mathrm{~A}$ & 121.00 \\
\hline $\mathrm{C} 3 \mathrm{~B}-\mathrm{C} 4 \mathrm{~B}-\mathrm{C} 5 \mathrm{~B}$ & $122.5(6)$ & $\mathrm{C} 9 \mathrm{~A}-\mathrm{C} 4 \mathrm{~A}-\mathrm{H} 4 \mathrm{~A}$ & 121.00 \\
\hline $\mathrm{C} 4 \mathrm{~B}-\mathrm{C} 5 \mathrm{~B}-\mathrm{C} 6 \mathrm{~B}$ & $119.5(6)$ & $\mathrm{C} 4 \mathrm{~A}-\mathrm{C} 5 \mathrm{~A}-\mathrm{H} 5 \mathrm{~A}$ & 119.00 \\
\hline $\mathrm{C} 1 \mathrm{~B}-\mathrm{C} 6 \mathrm{~B}-\mathrm{C} 5 \mathrm{~B}$ & $120.9(7)$ & $\mathrm{C} 6 \mathrm{~A}-\mathrm{C} 5 \mathrm{~A}-\mathrm{H} 5 \mathrm{~A}$ & 119.00 \\
\hline $\mathrm{O} 11 \mathrm{~B}-\mathrm{C} 12 \mathrm{~B}-\mathrm{C} 13 \mathrm{~B}$ & $112.9(5)$ & $\mathrm{C} 5 \mathrm{~A}-\mathrm{C} 6 \mathrm{~A}-\mathrm{H} 6 \mathrm{~A}$ & 119.00 \\
\hline $\mathrm{O} 13 \mathrm{~B}-\mathrm{C} 13 \mathrm{~B}-\mathrm{O} 14 \mathrm{~B}$ & $127.8(5)$ & $\mathrm{C} 7 \mathrm{~A}-\mathrm{C} 6 \mathrm{~A}-\mathrm{H} 6 \mathrm{~A}$ & 119.00 \\
\hline $\mathrm{O} 13 \mathrm{~B}-\mathrm{C} 13 \mathrm{~B}-\mathrm{C} 12 \mathrm{~B}$ & $117.9(5)$ & $\mathrm{C} 6 \mathrm{~A}-\mathrm{C} 7 \mathrm{~A}-\mathrm{H} 7 \mathrm{~A}$ & 121.00 \\
\hline $\mathrm{O} 14 \mathrm{~B}-\mathrm{C} 13 \mathrm{~B}-\mathrm{C} 12 \mathrm{~B}$ & $114.0(5)$ & $\mathrm{C} 8 \mathrm{~A}-\mathrm{C} 7 \mathrm{~A}-\mathrm{H} 7 \mathrm{~A}$ & 121.00 \\
\hline $\mathrm{C} 2 \mathrm{~B}-\mathrm{C} 3 \mathrm{~B}-\mathrm{H} 3 \mathrm{~B}$ & 122.00 & $\mathrm{C} 3 \mathrm{~A}-\mathrm{C} 31 \mathrm{~A}-\mathrm{H} 30 \mathrm{~B}$ & 108.00 \\
\hline
\end{tabular}




\begin{tabular}{|c|c|}
\hline $\mathrm{C} 4 \mathrm{~B}-\mathrm{C} 3 \mathrm{~B}-\mathrm{H} 3 \mathrm{~B}$ & 122.00 \\
\hline $\mathrm{C} 6 \mathrm{~B}-\mathrm{C} 5 \mathrm{~B}-\mathrm{H} 5 \mathrm{~B}$ & 120.00 \\
\hline $\mathrm{C} 4 \mathrm{~B}-\mathrm{C} 5 \mathrm{~B}-\mathrm{H} 5 \mathrm{~B}$ & 120.00 \\
\hline $\mathrm{C} 1 \mathrm{~B}-\mathrm{C} 6 \mathrm{~B}-\mathrm{H} 6 \mathrm{~B}$ & 120.00 \\
\hline $\mathrm{C} 5 \mathrm{~B}-\mathrm{C} 6 \mathrm{~B}-\mathrm{H} 6 \mathrm{~B}$ & 120.00 \\
\hline $\mathrm{O} 11 \mathrm{~B}-\mathrm{C} 12 \mathrm{~B}-\mathrm{H} 13 \mathrm{~B}$ & 109.00 \\
\hline $\mathrm{C} 13 \mathrm{~B}-\mathrm{C} 12 \mathrm{~B}-\mathrm{H} 13 \mathrm{~B}$ & 109.00 \\
\hline $\mathrm{H} 12 \mathrm{~B}-\mathrm{C} 12 \mathrm{~B}-\mathrm{H} 13 \mathrm{~B}$ & 108.00 \\
\hline $\mathrm{C} 13 \mathrm{~B}-\mathrm{C} 12 \mathrm{~B}-\mathrm{H} 12 \mathrm{~B}$ & 109.00 \\
\hline $\mathrm{O} 11 \mathrm{~B}-\mathrm{C} 12 \mathrm{~B}-\mathrm{H} 12 \mathrm{~B}$ & 109.00 \\
\hline $\mathrm{C} 12 \mathrm{~B}-\mathrm{O} 11 \mathrm{~B}-\mathrm{C} 1 \mathrm{~B}-\mathrm{C} 2 \mathrm{~B}$ & $-177.8(5)$ \\
\hline $\mathrm{C} 12 \mathrm{~B}-\mathrm{O} 11 \mathrm{~B}-\mathrm{C} 1 \mathrm{~B}-\mathrm{C} 6 \mathrm{~B}$ & $-0.5(9)$ \\
\hline $\mathrm{C} 1 \mathrm{~B}-\mathrm{O} 11 \mathrm{~B}-\mathrm{C} 12 \mathrm{~B}-\mathrm{C} 13 \mathrm{~B}$ & $81.2(6)$ \\
\hline $\mathrm{C} 8 \mathrm{~A}-\mathrm{N} 1 \mathrm{~A}-\mathrm{C} 2 \mathrm{~A}-\mathrm{C} 3 \mathrm{~A}$ & $0.9(6)$ \\
\hline $\mathrm{C} 2 \mathrm{~A}-\mathrm{N} 1 \mathrm{~A}-\mathrm{C} 8 \mathrm{~A}-\mathrm{C} 7 \mathrm{~A}$ & $-179.8(6)$ \\
\hline $\mathrm{C} 2 \mathrm{~A}-\mathrm{N} 1 \mathrm{~A}-\mathrm{C} 8 \mathrm{~A}-\mathrm{C} 9 \mathrm{~A}$ & $-1.7(6)$ \\
\hline $\mathrm{C} 6 \mathrm{~B}-\mathrm{C} 1 \mathrm{~B}-\mathrm{C} 2 \mathrm{~B}-\mathrm{C} 3 \mathrm{~B}$ & $2.3(10)$ \\
\hline $\mathrm{O} 11 \mathrm{~B}-\mathrm{C} 1 \mathrm{~B}-\mathrm{C} 6 \mathrm{~B}-\mathrm{C} 5 \mathrm{~B}$ & $178.8(6)$ \\
\hline $\mathrm{C} 2 \mathrm{~B}-\mathrm{C} 1 \mathrm{~B}-\mathrm{C} 6 \mathrm{~B}-\mathrm{C} 5 \mathrm{~B}$ & $-3.8(9)$ \\
\hline $\mathrm{O} 11 \mathrm{~B}-\mathrm{C} 1 \mathrm{~B}-\mathrm{C} 2 \mathrm{~B}-\mathrm{C} 3 \mathrm{~B}$ & $179.9(6)$ \\
\hline $\mathrm{C} 6 \mathrm{~B}-\mathrm{C} 1 \mathrm{~B}-\mathrm{C} 2 \mathrm{~B}-\mathrm{Cl} 2 \mathrm{~B}$ & $-178.9(5)$ \\
\hline $\mathrm{O} 11 \mathrm{~B}-\mathrm{C} 1 \mathrm{~B}-\mathrm{C} 2 \mathrm{~B}-\mathrm{Cl} 2 \mathrm{~B}$ & $-1.3(8)$ \\
\hline $\mathrm{Cl} 2 \mathrm{~B}-\mathrm{C} 2 \mathrm{~B}-\mathrm{C} 3 \mathrm{~B}-\mathrm{C} 4 \mathrm{~B}$ & $-179.1(5)$ \\
\hline $\mathrm{C} 1 \mathrm{~B}-\mathrm{C} 2 \mathrm{~B}-\mathrm{C} 3 \mathrm{~B}-\mathrm{C} 4 \mathrm{~B}$ & $-0.2(9)$ \\
\hline $\mathrm{C} 2 \mathrm{~B}-\mathrm{C} 3 \mathrm{~B}-\mathrm{C} 4 \mathrm{~B}-\mathrm{Cl} 4 \mathrm{~B}$ & $-178.9(5)$ \\
\hline $\mathrm{C} 2 \mathrm{~B}-\mathrm{C} 3 \mathrm{~B}-\mathrm{C} 4 \mathrm{~B}-\mathrm{C} 5 \mathrm{~B}$ & $-0.4(9)$ \\
\hline $\mathrm{C} 3 \mathrm{~B}-\mathrm{C} 4 \mathrm{~B}-\mathrm{C} 5 \mathrm{~B}-\mathrm{C} 6 \mathrm{~B}$ & $-1.1(10)$ \\
\hline $\mathrm{C} 14 \mathrm{~B}-\mathrm{C} 4 \mathrm{~B}-\mathrm{C} 5 \mathrm{~B}-\mathrm{C} 6 \mathrm{~B}$ & $177.3(5)$ \\
\hline $\mathrm{C} 4 \mathrm{~B}-\mathrm{C} 5 \mathrm{~B}-\mathrm{C} 6 \mathrm{~B}-\mathrm{C} 1 \mathrm{~B}$ & $3.4(10)$ \\
\hline $\mathrm{O} 11 \mathrm{~B}-\mathrm{C} 12 \mathrm{~B}-\mathrm{C} 13 \mathrm{~B}-\mathrm{O} 13 \mathrm{~B}$ & $-5.6(7)$ \\
\hline $\mathrm{O} 11 \mathrm{~B}-\mathrm{C} 12 \mathrm{~B}-\mathrm{C} 13 \mathrm{~B}-\mathrm{O} 14 \mathrm{~B}$ & $168.9(5)$ \\
\hline
\end{tabular}

$$
\begin{array}{ll}
\text { C3A-C31A-H31B } & 109.00 \\
\text { C32A-C31A-H30B } & 109.00 \\
\text { C32A-C31A-H31B } & 109.00 \\
\text { H30B-C31A-H31B } & 108.00 \\
\text { N32A-C32A-H32B } & 109.00 \\
\text { N32A-C32A-H33B } & 109.00 \\
\text { C31A-C32A-H32B } & 109.00 \\
\text { C31A-C32A-H33B } & 109.00 \\
\text { H32B }- \text { C32A-H33B } & 108.00
\end{array}
$$$$
\mathrm{N} 1 \mathrm{~A}-\mathrm{C} 2 \mathrm{~A}-\mathrm{C} 3 \mathrm{~A}-\mathrm{C} 9 \mathrm{~A} \quad 0.3(5)
$$$$
\mathrm{N} 1 \mathrm{~A}-\mathrm{C} 2 \mathrm{~A}-\mathrm{C} 3 \mathrm{~A}-\mathrm{C} 31 \mathrm{~A} \quad 178.5(5)
$$$$
\mathrm{C} 2 \mathrm{~A}-\mathrm{C} 3 \mathrm{~A}-\mathrm{C} 9 \mathrm{~A}-\mathrm{C} 4 \mathrm{~A} \quad-179.6(6)
$$$$
\mathrm{C} 2 \mathrm{~A}-\mathrm{C} 3 \mathrm{~A}-\mathrm{C} 9 \mathrm{~A}-\mathrm{C} 8 \mathrm{~A} \quad-1.3(5)
$$$$
\mathrm{C} 31 \mathrm{~A}-\mathrm{C} 3 \mathrm{~A}-\mathrm{C} 9 \mathrm{~A}-\mathrm{C} 4 \mathrm{~A} \quad 2.1(9)
$$$$
\mathrm{C} 31 \mathrm{~A}-\mathrm{C} 3 \mathrm{~A}-\mathrm{C} 9 \mathrm{~A}-\mathrm{C} 8 \mathrm{~A} \quad-179.6(5)
$$$$
\mathrm{C} 2 \mathrm{~A}-\mathrm{C} 3 \mathrm{~A}-\mathrm{C} 31 \mathrm{~A}-\mathrm{C} 32 \mathrm{~A}-113.1(5)
$$$$
\mathrm{C} 9 \mathrm{~A}-\mathrm{C} 3 \mathrm{~A}-\mathrm{C} 31 \mathrm{~A}-\mathrm{C} 32 \mathrm{~A} \quad 64.8(6)
$$$$
\mathrm{C} 9 \mathrm{~A}-\mathrm{C} 4 \mathrm{~A}-\mathrm{C} 5 \mathrm{~A}-\mathrm{C} 6 \mathrm{~A} \quad 0.7(9)
$$$$
\mathrm{C} 5 \mathrm{~A}-\mathrm{C} 4 \mathrm{~A}-\mathrm{C} 9 \mathrm{~A}-\mathrm{C} 3 \mathrm{~A} \quad 179.0(6)
$$$$
\mathrm{C} 5 \mathrm{~A}-\mathrm{C} 4 \mathrm{~A}-\mathrm{C} 9 \mathrm{~A}-\mathrm{C} 8 \mathrm{~A} \quad 0.8(8)
$$$$
\mathrm{C} 4 \mathrm{~A}-\mathrm{C} 5 \mathrm{~A}-\mathrm{C} 6 \mathrm{~A}-\mathrm{C} 7 \mathrm{~A} \quad-1.9(11)
$$$$
\mathrm{C} 5 \mathrm{~A}-\mathrm{C} 6 \mathrm{~A}-\mathrm{C} 7 \mathrm{~A}-\mathrm{C} 8 \mathrm{~A} \quad 1.5(10)
$$$$
\mathrm{C} 6 \mathrm{~A}-\mathrm{C} 7 \mathrm{~A}-\mathrm{C} 8 \mathrm{~A}-\mathrm{N} 1 \mathrm{~A} \quad 178.0(6)
$$$$
\mathrm{C} 6 \mathrm{~A}-\mathrm{C} 7 \mathrm{~A}-\mathrm{C} 8 \mathrm{~A}-\mathrm{C} 9 \mathrm{~A} \quad 0.1(9)
$$$$
\mathrm{N} 1 \mathrm{~A}-\mathrm{C} 8 \mathrm{~A}-\mathrm{C} 9 \mathrm{~A}-\mathrm{C} 3 \mathrm{~A} \quad 1.8(6)
$$$$
\mathrm{N} 1 \mathrm{~A}-\mathrm{C} 8 \mathrm{~A}-\mathrm{C} 9 \mathrm{~A}-\mathrm{C} 4 \mathrm{~A} \quad-179.5(5)
$$$$
\mathrm{C} 7 \mathrm{~A}-\mathrm{C} 8 \mathrm{~A}-\mathrm{C} 9 \mathrm{~A}-\mathrm{C} 3 \mathrm{~A} \quad-179.9(5)
$$$$
\mathrm{C} 7 \mathrm{~A}-\mathrm{C} 8 \mathrm{~A}-\mathrm{C} 9 \mathrm{~A}-\mathrm{C} 4 \mathrm{~A} \quad-1.2(8)
$$$$
\mathrm{C} 3 \mathrm{~A}-\mathrm{C} 31 \mathrm{~A}-\mathrm{C} 32 \mathrm{~A}-\mathrm{N} 32 \mathrm{~A} \quad 58.6(5)
$$

Hydrogen-bond geometry $\left(\AA,{ }^{\circ}\right)$

\begin{tabular}{lllll}
\hline$D-\mathrm{H} \cdots A$ & $D-\mathrm{H}$ & $\mathrm{H} \cdots A$ & $D \cdots A$ & $D-\mathrm{H} \cdots A$ \\
\hline $\mathrm{N} 1 A-\mathrm{H} 1 A \cdots \mathrm{O} 13 B^{\mathrm{i}}$ & $0.87(4)$ & $2.13(5)$ & $2.879(6)$ & $144(6)$ \\
$\mathrm{N} 32 A-\mathrm{H} 34 A \cdots \mathrm{O} 14 B^{\mathrm{ii}}$ & $0.89(4)$ & $1.89(4)$ & $2.782(6)$ & $175(2)$ \\
$\mathrm{N} 32 A-\mathrm{H} 35 A \cdots \mathrm{O} 13 B^{\mathrm{iii}}$ & $0.90(4)$ & $2.10(5)$ & $2.817(6)$ & $137(4)$ \\
$\mathrm{N} 32 A-\mathrm{H} 36 A \cdots \mathrm{O} 13 B$ & $0.89(3)$ & $2.57(4)$ & $3.231(6)$ & $132(4)$ \\
$\mathrm{N} 32 A-\mathrm{H} 36 A \cdots \mathrm{O} 14 B$ & $0.89(3)$ & $1.94(4)$ & $2.816(6)$ & $171(5)$ \\
$\mathrm{C} 2 A-\mathrm{H} 2 A \cdots \mathrm{O} 14 B^{\mathrm{iii}}$ & 0.95 & 2.53 & $3.336(6)$ & 142 \\
\hline
\end{tabular}

Symmetry codes: (i) $x, y+1, z$; (ii) $-x+1, y-1 / 2,-z+1$; (iii) $-x+1, y+1 / 2,-z+1$. 


\section{(II) 2-(1H-Indol-3-yl)ethanaminium (3,5-dichlorophenoxy)acetate}

Crystal data

$\mathrm{C}_{10} \mathrm{H}_{13} \mathrm{~N}_{2}^{+} \cdot \mathrm{C}_{8} \mathrm{H}_{5} \mathrm{Cl}_{2} \mathrm{O}_{3}^{-}$

$M_{r}=381.24$

Monoclinic, $P 2_{1}$

Hall symbol: $\mathrm{P} 2 \mathrm{yb}$

$a=9.5154(8) \AA$

$b=6.1951(5) \AA$

$c=15.3646(9) \AA$

$\beta=102.579(7)^{\circ}$

$V=883.99(12) \AA^{3}$

$Z=2$

\section{Data collection}

Oxford Diffraction Gemini-S CCD-detector diffractometer

Radiation source: fine-focus sealed tube

Graphite monochromator

Detector resolution: 16.077 pixels $\mathrm{mm}^{-1}$

$\omega$ scans

Absorption correction: multi-scan

(CrysAlis PRO; Agilent, 2013)

$T_{\min }=0.872, T_{\max }=0.980$

Refinement

Refinement on $F^{2}$

Least-squares matrix: full

$R\left[F^{2}>2 \sigma\left(F^{2}\right)\right]=0.042$

$w R\left(F^{2}\right)=0.105$

$S=1.08$

2800 reflections

238 parameters

5 restraints

Primary atom site location: structure-invariant direct methods

Secondary atom site location: difference Fourier map

\section{Special details}

Geometry. Bond distances, angles etc. have been calculated using the rounded fractional coordinates. All su's are estimated from the variances of the (full) variance-covariance matrix. The cell esds are taken into account in the estimation of distances, angles and torsion angles

Refinement. Refinement of $\mathrm{F}^{2}$ against ALL reflections. The weighted R-factor $\mathrm{wR}$ and goodness of fit $\mathrm{S}$ are based on $\mathrm{F}^{2}$, conventional R-factors $R$ are based on $F$, with $F$ set to zero for negative $F^{2}$. The threshold expression of $F^{2}>2 \operatorname{sigma}\left(F^{2}\right)$ is used only for calculating R-factors(gt) etc. and is not relevant to the choice of reflections for refinement. R-factors based on $\mathrm{F}^{2}$ are statistically about twice as large as those based on F, and R- factors based on ALL data will be even larger.

Fractional atomic coordinates and isotropic or equivalent isotropic displacement parameters $\left(\AA^{2}\right)$

\begin{tabular}{lllll}
\hline & $x$ & $y$ & $z$ & $U_{\text {iso }}{ }^{*} / U_{\text {eq }}$ \\
\hline Cl3B & $0.88773(13)$ & $1.0762(2)$ & $0.94414(6)$ & $0.0556(4)$ \\
C15B & $0.61601(10)$ & $0.46571(17)$ & $1.09183(5)$ & $0.0421(3)$ \\
O11B & $0.6022(3)$ & $0.4935(4)$ & $0.75745(13)$ & $0.0337(8)$
\end{tabular}

$F(000)=396$

$D_{\mathrm{x}}=1.432 \mathrm{Mg} \mathrm{m}^{-3}$

Mo $K \alpha$ radiation, $\lambda=0.71073 \AA$

Cell parameters from 1140 reflections

$\theta=3.9-28.2^{\circ}$

$T=200 \mathrm{~K}$

Needle, colourless

$0.50 \times 0.12 \times 0.06 \mathrm{~mm}$

3845 measured reflections

2800 independent reflections

2451 reflections with $I>2 \sigma(I)$

$R_{\text {int }}=0.027$

$\theta_{\max }=26.0^{\circ}, \theta_{\min }=3.1^{\circ}$

$h=-11 \rightarrow 7$$$
l=-18 \rightarrow 18
$$

Hydrogen site location: inferred from neighbouring sites

$\mathrm{H}$ atoms treated by a mixture of independent and constrained refinement

$w=1 /\left[\sigma^{2}\left(F_{\mathrm{o}}^{2}\right)+(0.0513 P)^{2}\right]$

where $P=\left(F_{\mathrm{o}}{ }^{2}+2 F_{\mathrm{c}}{ }^{2}\right) / 3$

$\Delta / \sigma)_{\max }=0.001$

$\Delta \rho_{\min }=-0.24 \mathrm{e} \AA^{-3}$

Absolute structure: Flack (1983)

Absolute structure parameter: 0.01 (7) 


\begin{tabular}{|c|c|c|c|c|}
\hline O13B & $0.5666(3)$ & $0.2827(4)$ & $0.60040(14)$ & $0.0269(7)$ \\
\hline O14B & 0.4608 (3) & 0.0059 (4) & $0.65216(15)$ & $0.0353(8)$ \\
\hline $\mathrm{C} 1 \mathrm{~B}$ & 0.6435 (3) & $0.5773(6)$ & 0.84115 (19) & $0.0266(10)$ \\
\hline $\mathrm{C} 2 \mathrm{~B}$ & $0.7290(4)$ & $0.7600(6)$ & $0.8496(2)$ & $0.0304(10)$ \\
\hline $\mathrm{C} 3 \mathrm{~B}$ & $0.7776(4)$ & $0.8496(6)$ & $0.9324(2)$ & $0.0301(11)$ \\
\hline C4B & $0.7436(4)$ & $0.7632(6)$ & $1.0088(2)$ & $0.0306(10)$ \\
\hline C5B & $0.6601(4)$ & $0.5807(7)$ & 0.99749 (19) & $0.0298(10)$ \\
\hline C6B & $0.6062(3)$ & $0.4868(6)$ & $0.91596(19)$ & $0.0279(10)$ \\
\hline $\mathrm{C} 12 \mathrm{~B}$ & $0.5268(4)$ & $0.2927(6)$ & $0.7495(2)$ & $0.0297(11)$ \\
\hline C13B & $0.5178(3)$ & 0.1909 (5) & $0.6593(2)$ & $0.0243(10)$ \\
\hline N1A & $0.2443(3)$ & $0.6837(5)$ & $0.6332(2)$ & $0.0339(10)$ \\
\hline $\mathrm{N} 32 \mathrm{~A}$ & $0.3860(3)$ & $0.2111(5)$ & $0.42680(18)$ & $0.0238(8)$ \\
\hline $\mathrm{C} 2 \mathrm{~A}$ & $0.2536(3)$ & $0.5745(7)$ & $0.5567(2)$ & $0.0314(11)$ \\
\hline C3A & $0.1828(3)$ & $0.3819(6)$ & $0.5518(2)$ & $0.0238(10)$ \\
\hline $\mathrm{C} 4 \mathrm{~A}$ & $0.0375(4)$ & $0.2219(7)$ & $0.6612(2)$ & $0.0366(11)$ \\
\hline C5A & $-0.0092(4)$ & $0.2656(7)$ & $0.7385(2)$ & $0.0435(14)$ \\
\hline C6A & 0.0307 (4) & $0.4575(8)$ & $0.7856(2)$ & $0.0420(13)$ \\
\hline C7A & $0.1174(4)$ & 0.6077 (7) & $0.7582(2)$ & $0.0366(11)$ \\
\hline C8A & $0.1640(3)$ & $0.5635(6)$ & $0.6792(2)$ & $0.0293(10)$ \\
\hline C9A & $0.1237(3)$ & $0.3729(6)$ & $0.6306(2)$ & $0.0258(10)$ \\
\hline C31A & 0.1548 (4) & $0.2190(6)$ & $0.4786(2)$ & 0.0307 (11) \\
\hline $\mathrm{C} 32 \mathrm{~A}$ & $0.2298(3)$ & $0.2630(6)$ & $0.4028(2)$ & $0.0290(10)$ \\
\hline $\mathrm{H} 2 \mathrm{~B}$ & 0.75380 & 0.82280 & 0.79850 & $0.0360 *$ \\
\hline H4B & 0.77660 & 0.82720 & 1.06580 & $0.0370^{*}$ \\
\hline H6B & 0.54530 & 0.36380 & 0.91080 & $0.0340^{*}$ \\
\hline H12B & 0.42830 & 0.31680 & 0.75890 & $0.0350^{*}$ \\
\hline H13B & 0.57670 & 0.19270 & 0.79650 & $0.0350^{*}$ \\
\hline H1A & $0.291(4)$ & $0.803(5)$ & 0.649 (3) & $0.0530^{*}$ \\
\hline $\mathrm{H} 2 \mathrm{~A}$ & 0.30260 & 0.62610 & 0.51320 & $0.0380^{*}$ \\
\hline $\mathrm{H} 4 \mathrm{~A}$ & 0.01130 & 0.09110 & 0.62960 & $0.0440 *$ \\
\hline $\mathrm{H} 5 \mathrm{~A}$ & -0.06890 & 0.16460 & 0.75980 & $0.0530^{*}$ \\
\hline H6A & -0.00350 & 0.48420 & 0.83830 & $0.0500^{*}$ \\
\hline H7A & 0.14490 & 0.73640 & 0.79120 & $0.0440^{*}$ \\
\hline $\mathrm{H} 30 \mathrm{~A}$ & 0.04970 & 0.21190 & 0.45400 & $0.0370 *$ \\
\hline $\mathrm{H} 31 \mathrm{~A}$ & 0.18580 & 0.07560 & 0.50430 & $0.0370^{*}$ \\
\hline $\mathrm{H} 32 \mathrm{~A}$ & 0.18350 & 0.17590 & 0.35030 & $0.0350^{*}$ \\
\hline $\mathrm{H} 33 \mathrm{~A}$ & 0.21760 & 0.41720 & 0.38590 & $0.0350^{*}$ \\
\hline $\mathrm{H} 34 \mathrm{~A}$ & $0.420(4)$ & $0.237(7)$ & $0.4830(14)$ & $0.0530^{*}$ \\
\hline $\mathrm{H} 35 \mathrm{~A}$ & 0.407 (4) & $0.080(4)$ & 0.409 (3) & $0.0530^{*}$ \\
\hline $\mathrm{H} 36 \mathrm{~A}$ & $0.438(4)$ & $0.292(7)$ & 0.398 & $0.0530^{*}$ \\
\hline
\end{tabular}

Atomic displacement parameters $\left(\AA^{2}\right)$

\begin{tabular}{lllllll}
\hline & $U^{11}$ & $U^{22}$ & $U^{33}$ & $U^{12}$ & $U^{13}$ & $U^{23}$ \\
\hline Cl3B & $0.0674(7)$ & $0.0572(7)$ & $0.0408(5)$ & $-0.0354(6)$ & $0.0090(5)$ & $-0.0097(5)$ \\
C15B & $0.0558(6)$ & $0.0482(6)$ & $0.0235(4)$ & $-0.0057(5)$ & $0.0112(4)$ & $0.0014(4)$ \\
O11B & $0.0576(15)$ & $0.0254(14)$ & $0.0187(10)$ & $-0.0111(13)$ & $0.0099(10)$ & $-0.0035(10)$ \\
O13B & $0.0352(13)$ & $0.0241(13)$ & $0.0215(11)$ & $-0.0037(11)$ & $0.0061(10)$ & $-0.0029(10)$
\end{tabular}


supporting information

$\begin{array}{lllllll}\text { O14B } & 0.0435(14) & 0.0235(15) & 0.0423(13) & -0.0097(12) & 0.0168(11) & -0.0078(11) \\ \text { C1B } & 0.0358(18) & 0.0236(18) & 0.0205(15) & -0.0006(16) & 0.0066(14) & -0.0050(15) \\ \text { C2B } & 0.0355(18) & 0.032(2) & 0.0243(16) & -0.0005(17) & 0.0081(15) & 0.0001(16) \\ \text { C3B } & 0.0319(19) & 0.027(2) & 0.0300(17) & -0.0034(16) & 0.0038(16) & -0.0043(16) \\ \text { C4B } & 0.0289(17) & 0.037(2) & 0.0240(16) & -0.0029(16) & 0.0015(14) & -0.0103(16) \\ \text { C5B } & 0.0341(18) & 0.034(2) & 0.0215(15) & 0.0066(18) & 0.0066(13) & 0.0039(16) \\ \text { C6B } & 0.0336(17) & 0.0249(19) & 0.0251(15) & -0.0020(16) & 0.0059(14) & 0.0008(15) \\ \text { C12B } & 0.042(2) & 0.024(2) & 0.0236(16) & -0.0046(17) & 0.0085(15) & -0.0044(15) \\ \text { C13B } & 0.0266(17) & 0.0200(19) & 0.0257(15) & -0.0023(16) & 0.0041(14) & 0.0012(15) \\ \text { N1A } & 0.0349(17) & 0.0248(17) & 0.0427(16) & -0.0066(14) & 0.0099(14) & -0.0069(15) \\ \text { N32A } & 0.0264(14) & 0.0230(17) & 0.0216(12) & 0.0016(13) & 0.0044(12) & 0.0005(13) \\ \text { C2A } & 0.0261(17) & 0.033(2) & 0.0366(18) & -0.0040(17) & 0.0103(14) & -0.0023(17) \\ \text { C3A } & 0.0202(16) & 0.0249(19) & 0.0268(16) & -0.0021(14) & 0.0064(14) & 0.0008(15) \\ \text { C4A } & 0.041(2) & 0.034(2) & 0.0362(18) & -0.0090(19) & 0.0115(17) & 0.0043(17) \\ \text { C5A } & 0.044(2) & 0.052(3) & 0.038(2) & -0.008(2) & 0.0163(19) & 0.007(2) \\ \text { C6A } & 0.040(2) & 0.061(3) & 0.0260(16) & 0.009(2) & 0.0092(16) & 0.004(2) \\ \text { C7A } & 0.0336(19) & 0.044(2) & 0.0280(17) & 0.0066(18) & -0.0026(15) & -0.0081(18) \\ \text { C8A } & 0.0212(16) & 0.035(2) & 0.0297(17) & 0.0061(16) & 0.0010(14) & 0.0006(17) \\ \text { C9A } & 0.0213(16) & 0.028(2) & 0.0262(16) & 0.0012(15) & 0.0012(14) & 0.0018(15) \\ \text { C31A } & 0.0281(17) & 0.029(2) & 0.0348(18) & -0.0045(16) & 0.0062(15) & -0.0042(16) \\ \text { C32A } & 0.0267(17) & 0.032(2) & 0.0269(17) & 0.0044(16) & 0.0027(15) & -0.0013(16)\end{array}$

Geometric parameters $\left(\AA,{ }^{\circ}\right)$

\begin{tabular}{llll}
\hline $\mathrm{C} 13 \mathrm{~B}-\mathrm{C} 3 \mathrm{~B}$ & $1.738(4)$ & $\mathrm{C} 6 \mathrm{~B}-\mathrm{H} 6 \mathrm{~B}$ & 0.9500 \\
$\mathrm{C} 15 \mathrm{~B}-\mathrm{C} 5 \mathrm{~B}$ & $1.746(3)$ & $\mathrm{C} 12 \mathrm{~B}-\mathrm{H} 13 \mathrm{~B}$ & 0.9900 \\
$\mathrm{O} 11 \mathrm{~B}-\mathrm{C} 1 \mathrm{~B}$ & $1.363(4)$ & $\mathrm{C} 12 \mathrm{~B}-\mathrm{H} 12 \mathrm{~B}$ & 0.9900 \\
$\mathrm{O} 11 \mathrm{~B}-\mathrm{C} 12 \mathrm{~B}$ & $1.428(5)$ & $\mathrm{C} 2 \mathrm{~A}-\mathrm{C} 3 \mathrm{~A}$ & $1.364(5)$ \\
$\mathrm{O} 13 \mathrm{~B}-\mathrm{C} 13 \mathrm{~B}$ & $1.241(4)$ & $\mathrm{C} 3 \mathrm{~A}-\mathrm{C} 9 \mathrm{~A}$ & $1.443(4)$ \\
$\mathrm{O} 14 \mathrm{~B}-\mathrm{C} 13 \mathrm{~B}$ & $1.263(4)$ & $\mathrm{C} 3 \mathrm{~A}-\mathrm{C} 31 \mathrm{~A}$ & $1.491(5)$ \\
$\mathrm{N} 1 \mathrm{~A}-\mathrm{C} 8 \mathrm{~A}$ & $1.369(4)$ & $\mathrm{C} 4 \mathrm{~A}-\mathrm{C} 5 \mathrm{~A}$ & $1.383(5)$ \\
$\mathrm{N} 1 \mathrm{~A}-\mathrm{C} 2 \mathrm{~A}$ & $1.376(5)$ & $\mathrm{C} 4 \mathrm{~A}-\mathrm{C} 9 \mathrm{~A}$ & $1.392(5)$ \\
$\mathrm{N} 32 \mathrm{~A}-\mathrm{C} 32 \mathrm{~A}$ & $1.487(4)$ & $\mathrm{C} 5 \mathrm{~A}-\mathrm{C} 6 \mathrm{~A}$ & $1.401(6)$ \\
$\mathrm{N} 1 \mathrm{~A}-\mathrm{H} 1 \mathrm{~A}$ & $0.87(4)$ & $\mathrm{C} 6 \mathrm{~A}-\mathrm{C} 7 \mathrm{~A}$ & $1.369(6)$ \\
$\mathrm{N} 32 \mathrm{~A}-\mathrm{H} 34 \mathrm{~A}$ & $0.87(2)$ & $\mathrm{C} 7 \mathrm{~A}-\mathrm{C} 8 \mathrm{~A}$ & $1.407(4)$ \\
$\mathrm{N} 32 \mathrm{~A}-\mathrm{H} 36 \mathrm{~A}$ & $0.89(4)$ & $\mathrm{C} 8 \mathrm{~A}-\mathrm{C} 9 \mathrm{~A}$ & $1.405(5)$ \\
$\mathrm{N} 32 \mathrm{~A}-\mathrm{H} 35 \mathrm{~A}$ & $0.89(3)$ & $\mathrm{C} 31 \mathrm{~A}-\mathrm{C} 32 \mathrm{~A}$ & $1.517(5)$ \\
$\mathrm{C} 1 \mathrm{~B}-\mathrm{C} 2 \mathrm{~B}$ & $1.383(5)$ & $\mathrm{C} 2 \mathrm{~A}-\mathrm{H} 2 \mathrm{~A}$ & 0.9500 \\
$\mathrm{C} 1 \mathrm{~B}-\mathrm{C} 6 \mathrm{~B}$ & $1.393(4)$ & $\mathrm{C} 4 \mathrm{~A}-\mathrm{H} 4 \mathrm{~A}$ & 0.9500 \\
$\mathrm{C} 2 \mathrm{~B}-\mathrm{C} 3 \mathrm{~B}$ & $1.373(4)$ & $\mathrm{C} 5 \mathrm{~A}-\mathrm{H} 5 \mathrm{~A}$ & 0.9500 \\
$\mathrm{C} 3 \mathrm{~B}-\mathrm{C} 4 \mathrm{~B}$ & $1.391(5)$ & $\mathrm{C} 6 \mathrm{~A}-\mathrm{H} 6 \mathrm{~A}$ & 0.9500 \\
$\mathrm{C} 4 \mathrm{~B}-\mathrm{C} 5 \mathrm{~B}$ & $1.371(6)$ & $\mathrm{C} 7 \mathrm{~A}-\mathrm{H} 7 \mathrm{~A}$ & 0.9500 \\
$\mathrm{C} 5 \mathrm{~B}-\mathrm{C} 6 \mathrm{~B}$ & $1.375(4)$ & $\mathrm{C} 31 \mathrm{~A}-\mathrm{H} 30 \mathrm{~A}$ & 0.9900 \\
$\mathrm{C} 12 \mathrm{~B}-\mathrm{C} 13 \mathrm{~B}$ & $1.508(4)$ & $\mathrm{C} 31 \mathrm{~A}-\mathrm{H} 31 \mathrm{~A}$ & 0.9900 \\
$\mathrm{C} 2 \mathrm{~B}-\mathrm{H} 2 \mathrm{~B}$ & 0.9500 & $\mathrm{C} 32 \mathrm{~A}-\mathrm{H} 32 \mathrm{~A}$ & 0.9900 \\
$\mathrm{C} 4 \mathrm{~B}-\mathrm{H} 4 \mathrm{~B}$ & 0.9500 & $\mathrm{C} 32 \mathrm{~A}-\mathrm{H} 33 \mathrm{~A}$ & 0.9900 \\
& & & $110.8(3)$ \\
$\mathrm{C} 1 \mathrm{~B}-\mathrm{O} 11 \mathrm{~B}-\mathrm{C} 12 \mathrm{~B}$ & $116.7(2)$ & $\mathrm{N} 1 \mathrm{~A}-\mathrm{C} 2 \mathrm{~A}-\mathrm{C} 3 \mathrm{~A}$ & \\
& & &
\end{tabular}




\begin{tabular}{|c|c|}
\hline $\mathrm{C} 2 \mathrm{~A}-\mathrm{N} 1 \mathrm{~A}-\mathrm{C} 8 \mathrm{~A}$ & $108.7(3)$ \\
\hline $\mathrm{C} 8 \mathrm{~A}-\mathrm{N} 1 \mathrm{~A}-\mathrm{H} 1 \mathrm{~A}$ & $129(3)$ \\
\hline $\mathrm{C} 2 \mathrm{~A}-\mathrm{N} 1 \mathrm{~A}-\mathrm{H} 1 \mathrm{~A}$ & $122(3)$ \\
\hline $\mathrm{C} 32 \mathrm{~A}-\mathrm{N} 32 \mathrm{~A}-\mathrm{H} 35 \mathrm{~A}$ & $114(3)$ \\
\hline $\mathrm{H} 34 \mathrm{~A}-\mathrm{N} 32 \mathrm{~A}-\mathrm{H} 36 \mathrm{~A}$ & $105(4)$ \\
\hline $\mathrm{C} 32 \mathrm{~A}-\mathrm{N} 32 \mathrm{~A}-\mathrm{H} 36 \mathrm{~A}$ & $113(3)$ \\
\hline $\mathrm{H} 34 \mathrm{~A}-\mathrm{N} 32 \mathrm{~A}-\mathrm{H} 35 \mathrm{~A}$ & $114(4)$ \\
\hline $\mathrm{C} 32 \mathrm{~A}-\mathrm{N} 32 \mathrm{~A}-\mathrm{H} 34 \mathrm{~A}$ & $110(3)$ \\
\hline $\mathrm{H} 35 \mathrm{~A}-\mathrm{N} 32 \mathrm{~A}-\mathrm{H} 36 \mathrm{~A}$ & $100(4)$ \\
\hline $\mathrm{O} 11 \mathrm{~B}-\mathrm{C} 1 \mathrm{~B}-\mathrm{C} 2 \mathrm{~B}$ & $116.3(3)$ \\
\hline $\mathrm{C} 2 \mathrm{~B}-\mathrm{C} 1 \mathrm{~B}-\mathrm{C} 6 \mathrm{~B}$ & $120.2(3)$ \\
\hline $\mathrm{O} 11 \mathrm{~B}-\mathrm{C} 1 \mathrm{~B}-\mathrm{C} 6 \mathrm{~B}$ & $123.5(3)$ \\
\hline $\mathrm{C} 1 \mathrm{~B}-\mathrm{C} 2 \mathrm{~B}-\mathrm{C} 3 \mathrm{~B}$ & $119.4(3)$ \\
\hline $\mathrm{C} 2 \mathrm{~B}-\mathrm{C} 3 \mathrm{~B}-\mathrm{C} 4 \mathrm{~B}$ & $122.2(3)$ \\
\hline $\mathrm{Cl} 3 \mathrm{~B}-\mathrm{C} 3 \mathrm{~B}-\mathrm{C} 4 \mathrm{~B}$ & $118.0(3)$ \\
\hline $\mathrm{Cl} 3 \mathrm{~B}-\mathrm{C} 3 \mathrm{~B}-\mathrm{C} 2 \mathrm{~B}$ & $119.8(3)$ \\
\hline $\mathrm{C} 3 \mathrm{~B}-\mathrm{C} 4 \mathrm{~B}-\mathrm{C} 5 \mathrm{~B}$ & $116.4(3)$ \\
\hline $\mathrm{Cl} 15 \mathrm{~B}-\mathrm{C} 5 \mathrm{~B}-\mathrm{C} 4 \mathrm{~B}$ & $117.9(2)$ \\
\hline $\mathrm{C} 15 \mathrm{~B}-\mathrm{C} 5 \mathrm{~B}-\mathrm{C} 6 \mathrm{~B}$ & $118.3(3)$ \\
\hline $\mathrm{C} 4 \mathrm{~B}-\mathrm{C} 5 \mathrm{~B}-\mathrm{C} 6 \mathrm{~B}$ & $123.7(3)$ \\
\hline $\mathrm{C} 1 \mathrm{~B}-\mathrm{C} 6 \mathrm{~B}-\mathrm{C} 5 \mathrm{~B}$ & $118.0(3)$ \\
\hline $\mathrm{O} 11 \mathrm{~B}-\mathrm{C} 12 \mathrm{~B}-\mathrm{C} 13 \mathrm{~B}$ & $111.7(3)$ \\
\hline $\mathrm{O} 13 \mathrm{~B}-\mathrm{C} 13 \mathrm{~B}-\mathrm{O} 14 \mathrm{~B}$ & $125.1(3)$ \\
\hline $\mathrm{O} 13 \mathrm{~B}-\mathrm{C} 13 \mathrm{~B}-\mathrm{C} 12 \mathrm{~B}$ & $121.5(3)$ \\
\hline $\mathrm{O} 14 \mathrm{~B}-\mathrm{C} 13 \mathrm{~B}-\mathrm{C} 12 \mathrm{~B}$ & $113.4(3)$ \\
\hline $\mathrm{C} 1 \mathrm{~B}-\mathrm{C} 2 \mathrm{~B}-\mathrm{H} 2 \mathrm{~B}$ & 120.00 \\
\hline $\mathrm{C} 3 \mathrm{~B}-\mathrm{C} 2 \mathrm{~B}-\mathrm{H} 2 \mathrm{~B}$ & 120.00 \\
\hline $\mathrm{C} 5 \mathrm{~B}-\mathrm{C} 4 \mathrm{~B}-\mathrm{H} 4 \mathrm{~B}$ & 122.00 \\
\hline $\mathrm{C} 3 \mathrm{~B}-\mathrm{C} 4 \mathrm{~B}-\mathrm{H} 4 \mathrm{~B}$ & 122.00 \\
\hline $\mathrm{C} 1 \mathrm{~B}-\mathrm{C} 6 \mathrm{~B}-\mathrm{H} 6 \mathrm{~B}$ & 121.00 \\
\hline $\mathrm{C} 5 \mathrm{~B}-\mathrm{C} 6 \mathrm{~B}-\mathrm{H} 6 \mathrm{~B}$ & 121.00 \\
\hline $\mathrm{O} 11 \mathrm{~B}-\mathrm{C} 12 \mathrm{~B}-\mathrm{H} 13 \mathrm{~B}$ & 109.00 \\
\hline $\mathrm{C} 13 \mathrm{~B}-\mathrm{C} 12 \mathrm{~B}-\mathrm{H} 13 \mathrm{~B}$ & 109.00 \\
\hline $\mathrm{H} 12 \mathrm{~B}-\mathrm{C} 12 \mathrm{~B}-\mathrm{H} 13 \mathrm{~B}$ & 108.00 \\
\hline $\mathrm{C} 13 \mathrm{~B}-\mathrm{C} 12 \mathrm{~B}-\mathrm{H} 12 \mathrm{~B}$ & 109.00 \\
\hline $\mathrm{O} 11 \mathrm{~B}-\mathrm{C} 12 \mathrm{~B}-\mathrm{H} 12 \mathrm{~B}$ & 109.00 \\
\hline $\mathrm{C} 12 \mathrm{~B}-\mathrm{O} 11 \mathrm{~B}-\mathrm{C} 1 \mathrm{~B}-\mathrm{C} 2 \mathrm{~B}$ & $173.6(3)$ \\
\hline $\mathrm{C} 12 \mathrm{~B}-\mathrm{O} 11 \mathrm{~B}-\mathrm{C} 1 \mathrm{~B}-\mathrm{C} 6 \mathrm{~B}$ & $-5.2(5)$ \\
\hline $\mathrm{C} 1 \mathrm{~B}-\mathrm{O} 11 \mathrm{~B}-\mathrm{C} 12 \mathrm{~B}-\mathrm{C} 13 \mathrm{~B}$ & $-166.5(3)$ \\
\hline $\mathrm{C} 2 \mathrm{~A}-\mathrm{N} 1 \mathrm{~A}-\mathrm{C} 8 \mathrm{~A}-\mathrm{C} 7 \mathrm{~A}$ & $-176.4(3)$ \\
\hline $\mathrm{C} 2 \mathrm{~A}-\mathrm{N} 1 \mathrm{~A}-\mathrm{C} 8 \mathrm{~A}-\mathrm{C} 9 \mathrm{~A}$ & $0.8(4)$ \\
\hline $\mathrm{C} 8 \mathrm{~A}-\mathrm{N} 1 \mathrm{~A}-\mathrm{C} 2 \mathrm{~A}-\mathrm{C} 3 \mathrm{~A}$ & $-1.0(4)$ \\
\hline $\mathrm{O} 11 \mathrm{~B}-\mathrm{C} 1 \mathrm{~B}-\mathrm{C} 6 \mathrm{~B}-\mathrm{C} 5 \mathrm{~B}$ & $177.0(3)$ \\
\hline $\mathrm{C} 2 \mathrm{~B}-\mathrm{C} 1 \mathrm{~B}-\mathrm{C} 6 \mathrm{~B}-\mathrm{C} 5 \mathrm{~B}$ & $-1.8(5)$ \\
\hline $\mathrm{O} 11 \mathrm{~B}-\mathrm{C} 1 \mathrm{~B}-\mathrm{C} 2 \mathrm{~B}-\mathrm{C} 3 \mathrm{~B}$ & $-178.3(3)$ \\
\hline $\mathrm{C} 6 \mathrm{~B}-\mathrm{C} 1 \mathrm{~B}-\mathrm{C} 2 \mathrm{~B}-\mathrm{C} 3 \mathrm{~B}$ & $0.6(5)$ \\
\hline $\mathrm{C} 1 \mathrm{~B}-\mathrm{C} 2 \mathrm{~B}-\mathrm{C} 3 \mathrm{~B}-\mathrm{Cl} 3 \mathrm{~B}$ & $178.8(3)$ \\
\hline
\end{tabular}

$\begin{array}{ll}\mathrm{C} 2 \mathrm{~A}-\mathrm{C} 3 \mathrm{~A}-\mathrm{C} 9 \mathrm{~A} & 105.4(3) \\ \mathrm{C} 2 \mathrm{~A}-\mathrm{C} 3 \mathrm{~A}-\mathrm{C} 31 \mathrm{~A} & 129.7(3) \\ \mathrm{C} 9 \mathrm{~A}-\mathrm{C} 3 \mathrm{~A}-\mathrm{C} 31 \mathrm{~A} & 124.6(3) \\ \mathrm{C} 5 \mathrm{~A}-\mathrm{C} 4 \mathrm{~A}-\mathrm{C} 9 \mathrm{~A} & 118.8(4) \\ \mathrm{C} 4 \mathrm{~A}-\mathrm{C} 5 \mathrm{~A}-\mathrm{C} 6 \mathrm{~A} & 120.6(4) \\ \text { C5A-C6A-C7A } & 122.1(3) \\ \text { C6A-C7A-C8A } & 117.2(4) \\ \text { N1A-C8A-C9A } & 107.5(3) \\ \text { C7A-C8A-C9A } & 121.4(3) \\ \text { N1A-C8A-C7A } & 131.0(3) \\ \text { C3A-C9A-C4A } & 132.5(3) \\ \text { C3A-C9A-C8A } & 107.6(3) \\ \text { C4A-C9A-C8A } & 119.9(3) \\ \text { C3A-C31A-C32A } & 114.9(3) \\ \text { N32A-C32A-C31A } & 112.5(3) \\ \text { N1A-C2A-H2A } & 125.00 \\ \text { C3A-C2A-H2A } & 125.00 \\ \text { C5A-C4A-H4A } & 121.00 \\ \text { C9A-C4A-H4A } & 121.00 \\ \text { C4A-C5A-H5A } & 120.00 \\ \text { C6A-C5A-H5A } & 120.00 \\ \text { C5A-C6A-H6A } & 119.00 \\ \text { C7A-C6A-H6A } & 119.00 \\ \text { C6A-C7A-H7A } & 121.00 \\ \text { C8A-C7A-H7A } & 121.00 \\ \text { C3A-C31A-H30A } & 109.00 \\ \text { C3A-C31A-H31A } & 109.00 \\ \text { C32A-C31A-H30A } & 109.00 \\ \text { C32A-C31A-H31A } & 109.00 \\ \text { H30A-C31A-H31A } & 107.00 \\ \text { N32A-C32A-H32A } & 109.00 \\ \text { N32A-C32A-H33A } & 109.00 \\ \text { C31A-C32A-H32A } & 109.00 \\ \text { C31A-C32A-H33A } & 109.00 \\ \text { H32A-C32A-H33A } & 108.00 \\ & \\ & \end{array}$

$\begin{array}{ll}\mathrm{N} 1 \mathrm{~A}-\mathrm{C} 2 \mathrm{~A}-\mathrm{C} 3 \mathrm{~A}-\mathrm{C} 9 \mathrm{~A} & 0.8(4) \\ \mathrm{N} 1 \mathrm{~A}-\mathrm{C} 2 \mathrm{~A}-\mathrm{C} 3 \mathrm{~A}-\mathrm{C} 31 \mathrm{~A} & 174.8(3) \\ \mathrm{C} 2 \mathrm{~A}-\mathrm{C} 3 \mathrm{~A}-\mathrm{C} 9 \mathrm{~A}-\mathrm{C} 4 \mathrm{~A} & 177.5(4) \\ \mathrm{C} 2 \mathrm{~A}-\mathrm{C} 3 \mathrm{~A}-\mathrm{C} 9 \mathrm{~A}-\mathrm{C} 8 \mathrm{~A} & -0.3(4) \\ \mathrm{C} 31 \mathrm{~A}-\mathrm{C} 3 \mathrm{~A}-\mathrm{C} 9 \mathrm{~A}-\mathrm{C} 4 \mathrm{~A} & 3.1(6) \\ \mathrm{C} 31 \mathrm{~A}-\mathrm{C} 3 \mathrm{~A}-\mathrm{C} 9 \mathrm{~A}-\mathrm{C} 8 \mathrm{~A} & -174.8(3) \\ \mathrm{C} 2 \mathrm{~A}-\mathrm{C} 3 \mathrm{~A}-\mathrm{C} 31 \mathrm{~A}-\mathrm{C} 32 \mathrm{~A} & 7.3(5) \\ \mathrm{C} 9 \mathrm{~A}-\mathrm{C} 3 \mathrm{~A}-\mathrm{C} 31 \mathrm{~A}-\mathrm{C} 32 \mathrm{~A} & -179.7(3) \\ \mathrm{C} 9 \mathrm{~A}-\mathrm{C} 4 \mathrm{~A}-\mathrm{C} 5 \mathrm{~A}-\mathrm{C} 6 \mathrm{~A} & -0.6(6) \\ \mathrm{C} 5 \mathrm{~A}-\mathrm{C} 4 \mathrm{~A}-\mathrm{C} 9 \mathrm{~A}-\mathrm{C} 3 \mathrm{~A} & -176.3(3) \\ \mathrm{C} 5 \mathrm{~A}-\mathrm{C} 4 \mathrm{~A}-\mathrm{C} 9 \mathrm{~A}-\mathrm{C} 8 \mathrm{~A} & 1.3(5)\end{array}$




$\begin{array}{llll}\mathrm{C} 1 \mathrm{~B}-\mathrm{C} 2 \mathrm{~B}-\mathrm{C} 3 \mathrm{~B}-\mathrm{C} 4 \mathrm{~B} & -0.1(6) & \mathrm{C} 4 \mathrm{~A}-\mathrm{C} 5 \mathrm{~A}-\mathrm{C} 6 \mathrm{~A}-\mathrm{C} 7 \mathrm{~A} & -0.5(6) \\ \mathrm{C} 13 \mathrm{~B}-\mathrm{C} 3 \mathrm{~B}-\mathrm{C} 4 \mathrm{~B}-\mathrm{C} 5 \mathrm{~B} & -178.1(3) & \mathrm{C} 5 \mathrm{~A}-\mathrm{C} 6 \mathrm{~A}-\mathrm{C} 7 \mathrm{~A}-\mathrm{C} 8 \mathrm{~A} & 0.9(6) \\ \mathrm{C} 2 \mathrm{~B}-\mathrm{C} 3 \mathrm{~B}-\mathrm{C} 4 \mathrm{~B}-\mathrm{C} 5 \mathrm{~B} & 0.9(6) & \mathrm{C} 6 \mathrm{~A}-\mathrm{C} 7 \mathrm{~A}-\mathrm{C} 8 \mathrm{~A}-\mathrm{N} 1 \mathrm{~A} & 176.6(4) \\ \mathrm{C} 3 \mathrm{~B}-\mathrm{C} 4 \mathrm{~B}-\mathrm{C} 5 \mathrm{~B}-\mathrm{C} 6 \mathrm{~B} & -2.3(6) & \mathrm{C} 6 \mathrm{~A}-\mathrm{C} 7 \mathrm{~A}-\mathrm{C} 8 \mathrm{~A}-\mathrm{C} 9 \mathrm{~A} & -0.2(5) \\ \mathrm{C} 3 \mathrm{~B}-\mathrm{C} 4 \mathrm{~B}-\mathrm{C} 5 \mathrm{~B}-\mathrm{C} 15 \mathrm{~B} & 179.5(3) & \mathrm{N} 1 \mathrm{~A}-\mathrm{C} 8 \mathrm{~A}-\mathrm{C} 9 \mathrm{~A}-\mathrm{C} 3 \mathrm{~A} & -0.3(4) \\ \mathrm{C} 15 \mathrm{~B}-\mathrm{C} 5 \mathrm{~B}-\mathrm{C} 6 \mathrm{~B}-\mathrm{C} 1 \mathrm{~B} & -179.1(3) & \mathrm{N} 1 \mathrm{~A}-\mathrm{C} 8 \mathrm{~A}-\mathrm{C} 9 \mathrm{~A}-\mathrm{C} 4 \mathrm{~A} & -178.4(3) \\ \mathrm{C} 4 \mathrm{~B}-\mathrm{C} 5 \mathrm{~B}-\mathrm{C} 6 \mathrm{~B}-\mathrm{C} 1 \mathrm{~B} & 2.7(6) & \mathrm{C} 7 \mathrm{~A}-\mathrm{C} 8 \mathrm{~A}-\mathrm{C} 9 \mathrm{~A}-\mathrm{C} 3 \mathrm{~A} & 177.2(3) \\ \mathrm{O} 11 \mathrm{~B}-\mathrm{C} 12 \mathrm{~B}-\mathrm{C} 13 \mathrm{~B}-\mathrm{O} 13 \mathrm{~B} & -2.9(5) & \mathrm{C} 7 \mathrm{~A}-\mathrm{C} 8 \mathrm{~A}-\mathrm{C} 9 \mathrm{~A}-\mathrm{C} 4 \mathrm{~A} & -0.9(5) \\ \mathrm{O} 11 \mathrm{~B}-\mathrm{C} 12 \mathrm{~B}-\mathrm{C} 13 \mathrm{~B}-\mathrm{O} 14 \mathrm{~B} & 175.3(3) & \mathrm{C} 3 \mathrm{~A}-\mathrm{C} 31 \mathrm{~A}-\mathrm{C} 32 \mathrm{~A}-\mathrm{N} 32 \mathrm{~A} & 75.7(4)\end{array}$

Hydrogen-bond geometry $\left(A,{ }^{\circ}\right)$

\begin{tabular}{lllll}
\hline$D-\mathrm{H}^{\cdots} A$ & $D-\mathrm{H}$ & $\mathrm{H} \cdots A$ & $D \cdots A$ & $D-\mathrm{H} \cdots A$ \\
\hline $\mathrm{N} 1 A-\mathrm{H} 1 A \cdots \mathrm{O} 14 B^{\mathrm{i}}$ & $0.87(4)$ & $2.04(4)$ & $2.838(4)$ & $152(4)$ \\
$\mathrm{N} 32 A-\mathrm{H} 34 A \cdots \mathrm{O} 13 B$ & $0.87(2)$ & $2.05(3)$ & $2.875(4)$ & $160(4)$ \\
$\mathrm{N} 32 A-\mathrm{H} 35 A \cdots \mathrm{O} 11 B^{\text {ii }}$ & $0.89(3)$ & $2.60(4)$ & $3.160(4)$ & $122(3)$ \\
$\mathrm{N} 32 A-\mathrm{H} 35 A \cdots \mathrm{O} 13 B^{\text {ii }}$ & $0.89(3)$ & $1.87(3)$ & $2.739(4)$ & $164(4)$ \\
$\mathrm{N} 32 A-\mathrm{H} 36 A \cdots \mathrm{O} 14 B^{\text {iii }}$ & $0.89(4)$ & $1.90(4)$ & $2.775(4)$ & $170(4)$ \\
$\mathrm{C} 2 A-\mathrm{H} 2 A \cdots \mathrm{O} 13 B^{\text {iii }}$ & 0.95 & 2.55 & $3.495(4)$ & 177 \\
\hline
\end{tabular}

Symmetry codes: (i) $x, y+1, z$; (ii) $-x+1, y-1 / 2,-z+1$; (iii) $-x+1, y+1 / 2,-z+1$. 